\title{
Towards the reproducible fabrication of homogeneous SERS substrates by Langmuir-Schaefer technique: a low cost and scalable approach for practical SERS based sensing applications
}

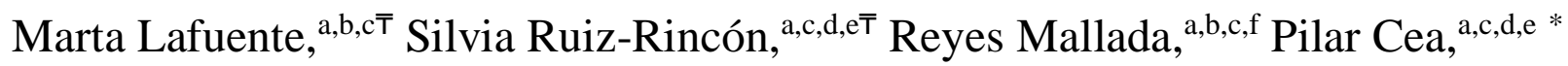 \\ and Maria Pilar Pina ${ }^{\mathrm{a}, \mathrm{b}, \mathrm{c}, \mathrm{f}}$ * \\ anstituto de Nanociencia de Aragón (INA). Campus Rio Ebro, Universidad de Zaragoza, C/Mariano Esquillor, s/n, \\ 50018 Zaragoza, Spain. \\ bDepartamento de Ingeniería Química y Tecnología del Medio Ambiente, Universidad de Zaragoza, 50009, Spain. \\ 'Instituto de Ciencia de Materiales de Aragón (ICMA), Universidad de Zaragoza-CSIC, 50009 Zaragoza, Spain. \\ ${ }^{\mathrm{d}}$ Departamento de Química Física, Facultad de Ciencias, Universidad de Zaragoza, 50009, Zaragoza, Spain. \\ 'Laboratorio de Microscopias Avanzadas (LMA). Campus Río Ebro, Universidad de Zaragoza, C/MarianoEsquilor, \\ s/n, 50018 Zaragoza, Spain. \\ ${ }^{\mathrm{f} N e t w o r k i n g ~ R e s e a r c h ~ C e n t e r ~ o n ~ B i o e n g i n e e r i n g, ~ B i o m a t e r i a l s ~ a n d ~ N a n o m e d i c i n e, ~ C I B E R-B B N, ~} 28029$ Madrid, \\ Spain \\ ${ }^{\top}$ These authors contributed equally to this work \\ * Corresponding authors: pcea@unizar.es (P.C.) and mapina@unizar.es (M.P.P.)
}

\section{Highlights:}

- Simple, reproducible and cost-effective fabrication of SERS substrates

- Octadecanethiol-capped Au NPs deposited using the Langmuir-Schaefer technique

- Homogenous coatings with a uniformly distributed SERS response over $\mathrm{cm}$ scale area

- Affordable and endurable SERS devices for routine high throughput analysis

\begin{abstract}
The development of homogeneous, endurable and low cost SERS (Surface Enhancement Raman Spectroscopy) substrates is becoming of outmost importance due to growing interest in SERS technique for analytical applications. Here, we use the Langmuir-Schaefer technique to produce, as demonstrated by Atomic Force Microscopy and Scanning Electron Microscopy, homogeneous tightly-packed films of $27 \mathrm{~nm}$ in diameter octadecylthiolate capped gold
\end{abstract}


nanoparticles in a reproducible and simple manner. The fabricated Au-based SERS substrates result in an analytical enhancement factor (AEF) of $4.3 \times 10^{8}$ and enable the detection of Rhodamine $6 \mathrm{G}$ in a $10^{-11} \mathrm{M}$ aqueous solution, with remarkable spatial uniformity of the SERS response over a scanned area of $0.4 \mathrm{~cm}^{2}$. In addition, the developed procedure is highly reproducible from batch to batch with a standard deviation below $10 \%$ in the SERS signal. Finally, these substrates exhibit durable SERS properties, i.e. experimentally validated 16 months after its fabrication.

Keywords: SERS substrates, Langmuir-Schaefer, solvent effect, analytical enhancement factor, spatial uniformity, pM detection

\section{Introduction}

Surface Enhanced Raman Spectroscopy (SERS) is one of the leading techniques for labelfree ultrasensitive vibrational fingerprinting of a variety of molecular compounds. The molecular specificity of Raman signal together with the high sensitivity of the SERS technique have made it a highly demanded analytical tool in various fields of chemistry, biology, medicine, genetics, forensics, pharmacy and environmental issues. For these reasons, academic and industrial interest in SERS has grown over the last decade as evidenced by the increasing number of publications involving the term "Surface Enhanced Raman", i.e. above 2,550 in 2018. Since its discovery in 1974 [1], the field of research is continuously evolving thanks to advances in bottom up and top-down strategies for the synthesis, assembly and fabrication of nanostructures combined with an increased understanding of their plasmonic properties.

By far, the SERS enhancement factor (EF) is the most commonly used parameter to quantify and compare the SERS activity of different substrates for practical applications [2, 3]. EF values varying from $10^{4}$ to $10^{12}$ may be found in the literature [4]. EF provides a quantitative indication of the enhancement of the Raman signal obtained with respect to the Raman response under non-SERS conditions for the same molecule in the same environment. The amplification of the Raman signal of molecules in SERS results from two main contributions: the electromagnetic mechanism provided by the localized surface plasmon resonance (LSPR) of metallic 
nanoparticles (NPs) or nanostructures under the excitation of light with suitable wavelengths and the chemical mechanism. Considerable efforts in the SERS field are focused on the development of methodologies for the processing of SERS substrates containing "hot spots", i.e. regions where the local field enhancement results in single molecule detection. The SERS performance depends on several parameters including composition, size, morphology, topology, surface distribution, and dielectric environment of the metallic NPs [5, 6]. Then, optimization of these parameters together with reliable and low cost methodologies for the preparation of homogenous SERS substrates is a field of major interest.

In general, the use of "top-down" lithography techniques to obtain well-defined periodic metallic nanostructures with tunable gaps leads to reliable SERS substrates with an exceptional control over the location, geometry, and density of hot spots [4]. However, manufacturing costs and production rates of these top-down methodologies are significant bottlenecks for large scale deployment. In an attempt for cost reduction, the generation of plasmonic nanogaps through the direct maskless dry etching of substrates combined with capillary leaning effects has been extensively studied [7, 8]. In spite thereof, the functionality of the nanostructured metallic surfaces is highly constrained when using the conventional cleanroom Si-based technologies. Indeed, cumbersome ad-hoc post-processing is mostly required for label-free SERS detection of target analytes.

On the other hand, "bottom-up" approaches open up a huge portfolio of potential SERS active substrates from the ordered assembly of metal colloids and NPs onto solid surfaces induced by mechanical or chemical processes. Since naked metal NPs are unstable in organic solvents, some form of stabilizer is required to prevent particle aggregation. Additionally, the presence of functional end groups within the ligand shell can have a profound influence on the physicochemical properties of the metal NPs. In fact, this ligand shell structure is often judiciously designed towards the specific adsorption of the target molecules when dealing with analytical SERS applications [9, 10]. Unlike top-down approaches, hot spots formation is not reproducible in bottom-up approaches due to facile aggregation of the plasmonic nanoparticles.

In general, the hot spots formation contributes significantly to the overall SERS signal, but the percentage of target molecules confined in such regions is negligible [11]. This fact 
jeopardizes the statistical relevance of the SERS measurements from an analytical point of view. This is the reason why the use of substrates with a more consistent, i.e. spatially homogenously distributed, yet lower average enhancement factors is becoming widespread for stable and reproducible SERS-based chemical sensing [12] in real-life situations [13].

The Langmuir-Blodgett (LB) and Langmuir-Schaeffer (LS) techniques represent one of the most sophisticated methods for the fabrication of well-ordered two-dimensional (2D) nanostructured materials. Although initially designed for the preparation of mono and multilayers of organic amphiphilic compounds [14], LB and LS techniques were later extended to the assembly of a wide variety of materials including metal NPs [15, 16]. These methodologies result, after appropriate optimization of certain parameters, in homogeneous and tightly-packed films. Additionally, these ordered assemblies can be deposited on virtually any substrate. These techniques also offer control on the distribution of the NPs and the surface concentration by modifying the surface pressure of transference. The method is also very convenient for deposition of films on large area substrates (over tens of $\mathrm{cm}^{2}$ ). Moreover, the production costs are relatively low since a Langmuir trough is operated in ambient conditions and from the technical point of view is a simple procedure. Examples of the use of the LB technique in the field of SERS substrates include the preparation of templates of organic functionalized materials onto which, in a subsequent incubation process (ca. 24 hours), noble metal NPs were deposited by self-assembly methodologies [17, 18]. Although strong evidences of efficient SERS sensing platforms were provided, this fabrication protocol has three limitations: (i) requires longer preparation times for the SERS substrate in comparison with just a simple LB or LS deposition of metallic NPs; (ii) results in relatively low surface coverages due to repulsion between the charged surface of the colloidal particles; and, (iii) the vibrational fingerprinting of the target molecules may be hampered by overlapping with the Raman signals of the organic templates. Tahghighi et al. [19] have recently prepared LB films incorporating commercial gold nanoparticles (AuNPs) but a good SERS performance required further modification of the film by using an electroless deposition processes. In fact, there are rather few examples of direct preparation of LB or LS films incorporating NPs without subsequent refining protocols to achieve a significant SERS enhancement. An important contribution in this context comes from Pérez-Mayen et al.[20] who described the preparation and transfer of LB films incorporating star-like AuNPs to Au-mirror configuration Si substrates for R6B detection. 
However, the AuNPs surface coverage, as evidenced from SEM images, still invites to improve the methodology for further enhancement of the spatial uniformity and surface density. These authors also evaluated the SERS performance of star-like AuNPs LB films transferred on Si substrates, attaining R6B detection in $10^{-9} \mathrm{M}$ concentration. Also remarkable is the work by Koh et al. [21] who fabricated a plasmonic nose based on silver nanocubes. This plasmonic nose device contained a zeolitic imidazolate framework partition layer to enhance the number of entrapped molecules of volatile organic compounds within the proximity of Ag particles. These authors used the LB technique to control the density of silver nanocubes on the surface in order to maximize the SERS signal.

Taking advantage of the LS technique, this contribution pursues the development of low cost substrates where the SERS signal results from target molecules settled at "cold sites" which constitute the vast majority of those exposed to the laser beam. Remarkably, reproducible fabrication of highly dense films incorporating thiol-capped AuNPs by the LS technique in one single step and over an area in the range of several $\mathrm{cm}^{2}$, for SERS based sensing applications has been attained by a comprehensive optimization of the preparation conditions. Thus, the hexane-chloroform ratio in the AuNPs suspension spread on the water surface of the Langmuir trough was extensively studied to identify the optimal solvent ratio. The SERS properties of the AuNPs-based substrates here fabricated have been evaluated by using R6G as probe molecule demonstrating the preparation of homogeneous, endurable, low-cost and large area SERS substrates, with a detection limit for R6G up to $10^{-11} \mathrm{M}$, i.e., higher than the current state of the art for other SERS substrates prepared by the LB or LS methodologies in one single step fabrication process over flat silicon [20]. Figure 1 summarizes the protocol followed for the fabrication and characterization of the SERS substrates presented in this paper.

\section{Materials and Methods}

\subsection{Materials}

$\mathrm{HAuCl}_{4} \cdot x \mathrm{H}_{2} \mathrm{O} \quad(\sim 50$ Au basis units), sodium citrate tribasic dehydrate (>99\%), 1octadecanethiol (98\%), Rhodamine 6G (R6G, 99\%), and hexamethyldisilazane (99.9\%) were purchased from Sigma-Aldrich. Acetone (99.5\%) and isopropyl alcohol (99.5\%) were provided 
by PanReac. Hexane (95\%) and chloroform (99\%) were supplied by Fisher Chemicals and Acros Organics, respectively. All solutions were prepared in Milli-pore MilliQ water (resistivity 18.2 $\mathrm{M} \Omega \cdot \mathrm{cm}$ ). 4" polished $\mathrm{SiO}_{2} / \mathrm{Si}$ wafers from Sil’Tronix (wet thermal oxide $1 \mu \mathrm{m}$ thick) were used as substrates onto which the Langmuir-Schaeffer films were transferred.

\subsection{Synthesis of AuNPs}

AuNPs were prepared using a method described previously.[9] Briefly, $\mathrm{HAuCl}_{4} \cdot x \mathrm{H}_{2} \mathrm{O}(19$ $\mathrm{mg}$ ) was dissolved in MilliQ water $(50 \mathrm{~mL})$ at $70^{\circ} \mathrm{C}$. Afterward, preheated sodium citrate $(5 \mathrm{~mL}$, $38 \times 10^{-3} \mathrm{M}$ ) was added dropwise to the first solution under magnetic stirring. The final solution was kept at $70{ }^{\circ} \mathrm{C}$ during $10 \mathrm{~min}$, showing a red-wine color. Then, the AuNPs dispersion was cooled to room temperature and stored at $4{ }^{\circ} \mathrm{C}$.

Octadecylthiolate protected AuNPs (AuNP-C18) were prepared at room temperature by a two-phase liquid-liquid system based on the method reported by Brust et al.[22] $15 \mathrm{~mL}$ of an aqueous solution of AuNPs were mixed with a solution of 1-octadecanethiol in hexane (7 $\mathrm{g}$ in 30 $\mathrm{mL}$ of hexane). The two-phase mixture was stirred vigorously until the AuNP-C 18 dispersion was transferred to the organic phase. The organic phase completely changed color from colorless to blue in 45 minutes (see Figure 1). Then, the mixture was added to a separating flask. The organic phase was kept and a fresh solution of 1-octadecanethiol $(1 \mathrm{~g}$ in $15 \mathrm{~mL}$ of hexane) was added to the aqueous phase. After stirring for $20 \mathrm{~min}$, the organic phase was separated again. All the organic solutions were mixed and centrifuged ( 3 cycles at 11,000 rpm during $15 \mathrm{~min}$ ) in order to remove the non-adsorbed octadecanethiol molecules. Finally, the centrifugalized pellet was redispersed into two different solvents (hexane and chloroform) and mixtures of them in several ratios. The final gold concentration in the AuNP-C 18 dispersion is $1.1 \mathrm{mg} \cdot \mathrm{mL}^{-1}$ as determined by the amount of solvent infused and assuming both: a yield of $100 \%$ in the ligand exchange process and no loss of material in the recovery process. In order to define the most suitable conditions for the fabrication of homogeneous and tightly-packed films of AuNP-C 18 , experimental studies were carried out under different hexane:chloroform volumetric ratios of the spreading solution, i.e. 1:0,1:1, 1:3, 1:5, 1:7 and 0:1. The dispersions of the gold nanoparticles were named as "AuNP-C 18 hexane:chloroform solvent ratio", for instance: AuNP-C 18_1:5 for AuNPs- $\mathrm{C}_{18}$ dispersed in a hexane:chloroform 1:5 solvent mixture. 


\subsection{Preparation of SERS substrates by Langmuir-Schaefer technique}

The preparation of SERS substrates by horizontal (Langmuir-Schaefer) transfer of the densely compressed AuNP-C 18 films is schematized in Figure 1. A 580 x $145 \mathrm{~mm}^{2} \mathrm{KSV}$ trough housed in a clean room at a constant temperature $\left(20 \pm 1^{\circ} \mathrm{C}\right)$ was used to prepare the Langmuir films and the Langmuir-Schaefer films. The surface pressure $(\pi)$ of the films was measured by means of a Wilhelmy paper plate pressure sensor. Milli-Q water was used as the subphase in the trough. $1.8 \mathrm{~mL}$ of a $1.1 \mathrm{mg} \cdot \mathrm{mL}^{-1}$ solution of AuNP-C 18 in hexane:chloroform were spread onto the water subphase by using a Hamilton microsyringe. Such initial low particle surface density avoids the irreversible formation of 3D metallic clusters. The spreading solvent or mixture of solvents was allowed to evaporate (over a period of at least $30 \mathrm{~min}$ ). After the solvent(s) evaporation, the film was compressed at a constant sweeping speed of $6 \mathrm{~mm} \cdot \mathrm{min}^{-1}$. The Langmuir films were transferred onto quartz for recording UV-vis spectra, and onto $\mathrm{SiO}_{2} / \mathrm{Si}$ substrates $(5 \mathrm{~mm} \times 8 \mathrm{~mm}$ ) for Atomic Force Microscopy (AFM), Scanning Electron Microscopy (SEM), and SERS experiments. Prior to the deposition process, the substrates were washed in ultrasound baths with acetone, isopropyl alcohol and Milli-Q water for $10 \mathrm{~min}$ in each one. They were subsequently dried under a $\mathrm{N}_{2}$ stream. Finally, the substrates were immersed in hexamethyldisilazane (99.9\%) for 24 hours to make the surface of the substrates hydrophobic, and dried at room conditions since it is well-known that hydrophobic surfaces promote the attachment of AuNPs covered with alkyl thiolates [20]. In all cases, the films were transferred at a constant surface pressure of $20 \mathrm{mN} \cdot \mathrm{m}^{-1}$ by the horizontal approach of the hydrophobic substrate to the aqueous subphase at a controlled speed of $1 \mathrm{~mm} \cdot \mathrm{min}^{-1}$.

\subsection{Raman and SERS Measurements}

All the vibrational experiments (SERS and Raman) were performed using an Alpha 300 Raman spectrometer (WITec) with a confocal optical microscope with a lateral resolution of 480 $\mathrm{nm}$. All measurements were made in backscattering geometry, using $20 \times$ microscope objective (scattering area of $1.86 \mu \mathrm{m}^{2}$ ), $633 \mathrm{~nm}$ as excitation laser line and $0.1 \mathrm{~mW}$ of incident power. A $10^{-3} \mathrm{M}$ stock solution of R6G in MilliQ was used to prepare further dilutions, $10^{-6} \mathrm{M}, 10^{-8} \mathrm{M}, 10^{-9}$ $\mathrm{M}$ and $10^{-11} \mathrm{M}$. SERS substrates were incubated in $1 \mathrm{~mL}$ of the adequate concentration of R6G in vertical position overnight; then, they were rinsed in MilliQ and air-dried. Between three to six 
different maps of $250 \times 150 \mu \mathrm{m}^{2}$ containing 225 excitations points were measured on the $\mathrm{SiO}_{2} / \mathrm{Si}$ substrates covered by the LS films. Acquisition conditions were varied as a function of the probe concentration, i.e., $1 \mathrm{~s}$ collection time for R6G $10^{-6} \mathrm{M}$ and $2 \mathrm{~s}$ collection time for less concentrated R6G solutions. The Raman intensity of C-C stretching vibrational mode at 1510 $\mathrm{cm}^{-1}$ was used as the mapped signal. In addition, normal Raman spectra were measured in a $10^{-3}$ M R6G solution spread on a PTFE dish, focusing the laser beam with a power of $15 \mathrm{~mW}$ and a collection time of $50 \mathrm{~s}$. Automatic baselines (background) have been subtracted by WITec Software 2.10 for all SERS and Raman spectra.

\subsection{Determination of analytical enhancement factor (AEF).}

Several methods have been reported to characterize the SERS properties of a plasmonic substrate [2]. Depending on the method, the reported enhancement factor may vary even a few orders of magnitude. In this work, we use the analytical enhancement factor (AEF) [2, 10, 23, 24]. The AEF provides information about the signal gain that one SERS substrate produces in comparison to a reference Raman experiment with the same molecule under almost identical experimental conditions (spectrophotometer, configuration, lenses, laser wavelength and power) and it is calculated as follows:

$$
\mathrm{AEF}=\frac{\mathrm{I}_{\text {SERS }} / \mathrm{C}_{\text {SERS }}}{\mathrm{I}_{\text {Raman }} / \mathrm{C}_{\text {Raman }}}
$$

where $C_{\text {Raman }}$ and $C_{S E R S}$ are the R6G concentration in the Raman measurements $(1 \mathrm{mM})$ and SERS conditions $(1 \mu \mathrm{M})$, respectively. And IRaman and ISERS are the normalized intensity values (cts. $\mathrm{mW}^{-1} \cdot \mathrm{s}^{-1}$ ) of the $\mathrm{R} 6 \mathrm{G}$ characteristic band at $1510 \mathrm{~cm}^{-1}$ for normal Raman and SERS measurements, respectively. ISERS was obtained from averaging more than 3,000 spectra.

\subsection{Material characterization}

UV-Vis spectra in liquid phase were obtained on a UV-Vis double beam spectrophotometer Jasco V670 using as reference the solvent of mixtures of solvents with the same solvent ratio of the AuNP-C 18 solution. Similarly, the UV-Vis absorption spectrum of the AuNP-C 18 LS film transferred onto quartz was recorded using a normal incident angle with respect to the film plane using a Varian Cary 50 spectrometer. 
Brewster Angle Microscopy (BAM) images of the Langmuir films were in situ recorded by using a commercial mini- BAM from Nanofilm Technologie GmbH, Göttingen, Germany. A commercial UV-Vis reflection spectrophotometer also from Nanofilm Technologie Gmb was employed to record the reflection spectra of the Langmuir films upon the compression process [25].

A Multimode 8 microscope equipped with a Nanoscope V control unit from Bruker was used to register Atomic Force Microscopy images. In particular, a scan rate of 1.0-1.2 Hz (512 lines) and force ranging $0.2-2 \mathrm{nN}$ were used by applying the Tapping mode. AFM data were obtained with RTESPA $\left(150 \mathrm{kHz}\right.$, and $3 \mathrm{~N} \cdot \mathrm{m}^{-1}$, from Bruker) tips. Images were typically recorded with scan rates of $1 \mathrm{~Hz} \cdot \mathrm{line}^{-1}$.

Analyses of TEM (recorded on carbon coated copper grids by FEI Tecnai T20) and SEM (FEG INSPECT 50) images were used to determine the size distribution and morphology of the AuNPs and to characterize the LS films, respectively.

\section{Results and discussion}

The use of alkanethiols as stabilizers provides a simple pathway for the transfer of AuNPs to organic solvents [22, 26, 27]. As described in the experimental section, octadecylthiolate protected gold nanoparticles, AuNP-C 18 , were prepared by means of a replacement of the original citrate stabilizer layer with 1-octadecanethiol. An excess of octadecanethiol was used to ensure a dense shell of stabilizer preventing particle aggregation. The average AuNP-C 18 size of the gold core is $22 \pm 9 \mathrm{~nm}$ as determined from statistical analysis of TEM images. TEM images of AuNP-citrate and AuNP-C18 (Figure S1 in the Supporting Information, SI) show that they maintain their particle size unaltered during the functionalization step. In addition, the organic shell is also distinguished in the AuNP-C 18 TEM images, with a thickness of $2.6 \pm 0.7 \mathrm{~nm}$. This value is in good agreement with a molecular model calculated using Spartan ${ }^{\circledR} 08$ V 1.0.0, that predicts a molecule length of $2.5 \mathrm{~nm}$ for the chemisorbed thiol on gold and a trans-zigzag conformation of the alkyl chain (see Figure S2 in the SI). Unlike AuNP-citrate in aqueous solution, showing the intense absorption line at $528 \mathrm{~nm}$, AuNP-C 18 hexane:chloroform dispersions exhibit a strong interaction between the metal surface and the organic binding agent 
through back $\pi$-bonding from the sulfur, resulting in dampening, broadening and large (red) shifting of the LSPR to $557 \mathrm{~nm}$ (Figure S3 in SI) [28].

Figure 2 shows the surface pressure vs. area $(\pi-A)$ isotherms for $\mathrm{AuNP}-\mathrm{C}_{18}$ dispersions in pure hexane (AuNP-C18_1:0), pure chloroform (AuNP-C $\left.18 \_0: 1\right)$ and hexane:chloroform in the 1:5 ratio (AuNP-C 18 1:5). An extension of this study using dispersions of AuNP-C 18 in mixtures of hexane:chloroform in several ratios can be found in Figure S4 in SI. The behavior of AuNP$\mathrm{C}_{18} \_1: 0$ sample clearly differs from the other dispersions. The slope of the $(\pi-A)$ isotherm for the AuNP-C $18 \_$1:0 sample decreases abruptly at ca. $15 \mathrm{mN} \cdot \mathrm{m}^{-1}$, with the isotherm exhibiting a plateau which is possibly indicating pilling up, NPs aggregation and 3D structures formation. On the contrary, for the AuNP-C 18 _0:1 sample, the surface pressure increases moderately in the region $500-1000 \mathrm{~nm}^{2} \cdot \mathrm{AuNP}^{-1}$ and then, sharply in the region $350-500 \mathrm{~nm}^{2} \cdot \mathrm{AuNP}^{-1}$ above 15 $\mathrm{mN} \cdot \mathrm{m}^{-1}$. The influence of the solvent nature on the final disposition of metal NPs prepared by the Langmuir-Blodgett or Langmuir-Schaefer techniques has been discussed previously in the literature [29]. On the one hand, a solvent with a high spreading coefficient is desirable since it favors the spreading of the material on the air-water interface. Hexane and chloroform have a spreading coefficient value at $20^{\circ} \mathrm{C}$ of 3.4 and $13.1 \mathrm{mN} \cdot \mathrm{m}^{-1}$ [30], respectively, which makes chloroform a more suitable spreading solvent. On the other hand, it is also known that long alkyl chains in the shell of the NPs tend to be extended in non-polar solvents such as hexane, which results in significant van der Waals forces among the alkyl chains of neighbor functionalized nanoparticles resulting in aggregation between the metal NPs, in agreement with the recorded $(\pi$ A) isotherm for AuNP-C18_1:0 sample [29]. In fact, AuNP-C18_1:0 dispersions are not stable and NPs tend to coagulate. On the contrary, the extension of the alkyl chains in a more polar solvent, such as chloroform, is less pronounced, resulting in weaker van der Waals interactions among neighbor gold NPs and thus, results in more stable dispersions.

From these statements one might think that chloroform is the ideal solvent to fabricate Langmuir films of AuNP-C 18 . However, our results (with experiments repeated for three times to ensure the reproducibility) show that a hexane:chloroform 1:5 ratio results in more homogeneous films that also exhibit a larger surface coverage. We attribute this behavior to a compromise at this hexane:chloroform 1:5 ratio for which the van der Waals forces among alkyl chains of neighbor AuNPs are weak enough to prevent coagulation (in solution) or collapse (at the air- 
water interface) of the AuNP-C 18 but strong enough to induce a close packing of the AuNP-C18. Similar densely packed films consisting of hydrophobic NPs have been described in the literature but using EG or DEG subphases or adding surfactants (PEG) to the water subphase to increase their affinity at the interface [28]. Additionally, Brewster Angle Microscopy images of AuNP$\mathrm{C}_{18}$ 1:5 Langmuir films (insets in Figure 2) show the formation of Langmuir films whose surface density increases progressively upon compression.

Reflection UV-vis spectra of the Langmuir films were recorded at several surface pressures upon compression and compared to the UV-vis spectrum obtained for the AuNPs dispersions. These results are illustrated in Figure 3, where only two reflection spectra are shown for clarifying purposes but the spectra for other surface pressures show an identical profile. Two relevant data are provided by these spectra. First, a significant red-shift, ca. $36 \mathrm{~nm}$, of the resonance plasmon band upon transference is observed: from the AuNP-C18_1:5 dispersion (at $557 \mathrm{~nm}$ ) to the AuNP-C18_1:5 in Langmuir films (at $593 \mathrm{~nm}$ ). This observation is consistent with the formation of AuNP-C 18 domains immediately after the spreading process on the aqueous subphase since it is well-known [31] that a red-shift of the plasmon peak in the spectrum of AuNPs is the response to visible light exhibited by collectives of AuNPs characterized by a short inter-particle distance. Most importantly, the position of the LSPR band in the AuNP-C18_1:5 Langmuir films remains constant with the compression process, i.e., no shift of the band is observed upon an increase in the surface density. The unchanged position of the plasmon band in the Langmuir films indicates that inter-particle spacing is preserved within the surface pressure range studied, i.e. from 1 to $20 \mathrm{mN} \cdot \mathrm{m}^{-1}$. This observation is attributable to the formation of ordered nanoparticle domains at the air-water interface from the beginning of the Langmuir film formation as a result of self-organization processes due to attractive forces between AuNP-C 18 as the solvent evaporates. As a result of the compression process, these domains get closer and closer, with an increase in the surface density that results in an increase in the reflection intensity values, and finally a well-ordered homogeneous AuNP-C18 film is formed. This argument is consistent with previous experimental and theoretical models of Langmuir films containing NPs $[19,28,32]$. Accounting from these results, Langmuir films of AuNP-C 18 dispersions in hexane:chloroform ratios were transferred at a constant surface pressure of $20 \mathrm{mN} \cdot \mathrm{m}^{-1}$. 
Figure 4 shows SEM images of these films. From these micrographs, which are reproducible over different areas of the same substrate and for different films prepared under identical conditions; it can be concluded that AuNP-C18_1:5 dispersion results in the most wellpacked and homogeneous AuNPs films from all the hexane:chloroform ratios herein studied.

The reproducibility in the protocol herein described can be assessed in Figure 5 that shows SEM images of four different LS films prepared from different dispersions of AuNP$\mathrm{C}_{18}$ 1:5. For all the samples, a homogenous tightly-packed arrangement of the particles on the surface is attained. Thus, the simple protocol here shown demonstrates consistent results and a high surface coverage of $\mathrm{Au}$ NPs, above 3100 AuNP-C 18 NPs $\cdot \mu \mathrm{m}^{-2}$ according to the $\pi-A$ isotherm (Figure 2).

A representative AFM image of LS films of AuNP-C18_1:5 deposited onto $\mathrm{SiO}_{2} / \mathrm{Si}$ substrates is shown in Figure 6.a. The AFM image shows a homogeneous and tightly-packed film as already demonstrated by SEM, with very few uncovered areas and other minor regions with bilayers or eventually multilayered films. The root mean square (RMS) roughness of the AuNPC18_1:5 film is $5.0 \mathrm{~nm}$. A section profile is also shown in Figure 6.b. The height of the film with respect to uncoated regions is in the $15-20 \mathrm{~nm}$ range. This value is consistent with the $27 \mathrm{~nm}$ (22 for the gold core and $2 \times 2.5 \mathrm{~nm}$ for the thiol shell) average value of the AuNP-C18 as determined from TEM, since immobilized particles onto a solid support tend to adopt a disk shape.[33] AFM images are also consistent with the large surface coverage observed in SEM images and estimated from the $(\pi-A)$ isotherm at $20 \mathrm{mN} \cdot \mathrm{m}^{-1}$. AFM phase images provide a clearer distinction between regions with a different chemical composition [34, 35]. The corresponding phase contrast image displayed in Figure 6.c shows in better detail the interparticle spacing due to interlocking between the aliphatic chains of adjacent nanoparticles in the 2D array.

The UV-vis absorption spectrum of the LS films transferred to the rigid substrate exhibits the same maximum wavelength value for the plasmon band, $593 \mathrm{~nm}$, as the Langmuir films (Figure 3). This result indicates that the spatial organization of the AuNP-C 18 in the Langmuir film is preserved upon the transference and drying process. 
In order to evaluate the SERS capabilities of AuNP-C $18 \_1: 5 \mathrm{LS}$ films on $\mathrm{SiO}_{2} / \mathrm{Si}$ chips (5 $\mathrm{mm} \times 8 \mathrm{~mm}$ ), the resulting Raman fingerprint of R6G probe from dilute solutions was determined. R6G was selected as a reference molecule since it has been widely used in literature for this purpose $[10,36]$ and will facilitate comparison with previously published data. The standard protocol includes the storage of the SERS substrates at room temperature in an air atmosphere and the incubation in the indicated solutions of R6G the day before measuring. The SERS spectra were acquired at an excitation wavelength of $633 \mathrm{~nm}$ for optimal coupling between SPR band and the incident laser line. The homogeneity of the SERS response was carefully analyzed. Figure 7.A shows the mapping of the R6G Raman signal at $1510 \mathrm{~cm}^{-1}$ and the average SERS spectra registered over extended areas $(250 \mu \mathrm{m} \times 150 \mu \mathrm{m}$ containing 225 excitations points) at three different locations (labeled as A1, A2, A3) within the same sample prepared from AuNP-C ${ }_{18} \_1: 5$. The average value and standard deviation for the SERS signal at $1510 \mathrm{~cm}^{-1}$ is $55784 \pm 6133 \mathrm{cts} \mathrm{mW}^{-1} \mathrm{~s}^{-1}$. This result demonstrates the huge potential of the LS method for the centimeter scale preparation of 2D arrays of AuNPs with remarkable SERS activity [36].

Additionally, the influence of the surface density of AuNP-C 18 on the SERS intensity was studied by comparing the response of LS films prepared from AuNP-C 18_1 $_{1}$ 7, AuNP-C 18_ $_{1}$ :5, and. AuNP-C18_1:3. As illustrated in Figure 7.B, the LS film incorporating AuNP-C 18 1:7 exhibits a SERS response with a significantly lower intensity (in comparison to the films prepared from AuNP-C $\left.18 \_1: 5\right)$, i.e., $22241 \pm 2156 \mathrm{cts} \mathrm{mW}^{-1} \mathrm{~s}^{-1}$ for the SERS signal at $1510 \mathrm{~cm}^{-1}$. Similarly, Figure S5 shows the SERS mappings for LS films prepared from AuNP-C18_1:3,

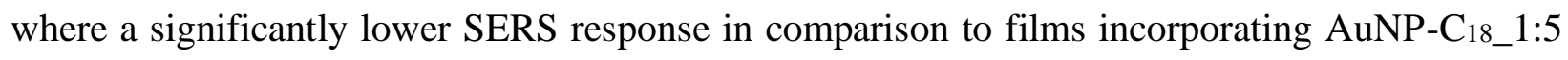
is obtained. The standard deviation of the SERS intensities recorded for a certain SERS mapping area is in the 27-30\%, 6-10\% and 23-46\% range for LS films incorporating AuNP-C 18 _ 1:7, AuNP-C $18 \_1: 5$ and AuNP-C $18 \_1: 3$, respectively. The lower spatial uniformity of the SERS response for AuNP-C 18_1 $_{-}$:7 and AuNP-C 18_1 $_{-} 1: 3$ films is attributed to changes in the surface density of the films, as evidenced by SEM images in Figure 4. It is well known that tightly packed films allow the short-range coupling between adjacent NPs increasing the local electromagnetic field and therefore, increasing the Raman signal. From averaging the above discussed mapping results, the analytical enhancement factor (AEF) is $1.4 \times 10^{8}, 4.3 \times 10^{8}$ and $1.7 \times 10^{8}$ in films incorporating AuNP-C $18 \_1: 3$, AuNP-C $_{18} \_1: 5$ and AuNP-C $18 \_1: 7$, respectively. 
To corroborate the enhanced ordering and surface density of the LS films herein investigated, we compare these results with those from a reference SERS substrate prepared by means of electrostatically interactions between citrate capped AuNPs onto a $\mathrm{SiO}_{2} / \mathrm{Si}$ surface previously modified with a cationic polyelectrolyte [9] (Figure S6). The intensity of the peak at $1510 \mathrm{~cm}^{-1}$ was treated statistically for each mapping. The average SERS intensity of this

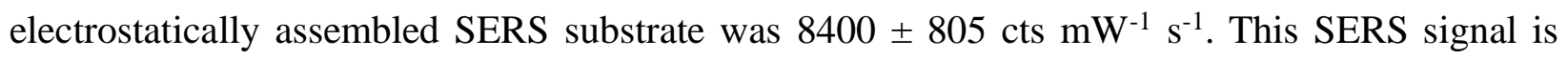
about 7-fold lower than the one from an AuNP-C 18 1:5 LS film $\left(55784 \pm 6133 \mathrm{cts} \mathrm{mW}^{-1} \mathrm{~s}^{-1}\right)$. Here, it is noteworthy that the 1-octadecanethiol stabilizer is less active in Raman than the citrate ligand. In fact, the octadecylthiolate capped Au NPs based substrates herein prepared soften the background signal, enabling a wider spectral window, which is really beneficial for potential applications on both qualitative and quantitative analysis.

Figure 8.a shows the SERS response of the AuNP-C $18 \_1: 5$ based substrates after incubation in R6G solutions of different concentrations. In order to analyze the potential ageing effect of the substrates, the SERS response of recently prepared and 16-month aged substrates was measured under equivalent conditions $\left(10^{-6} \mathrm{M}\right.$ solution of R6G). A similar average SERS intensity is obtained after 16 months of aging when compared with fresh samples (Figures 8.a and 8.b). This result supports the stability at room conditions of the AuNP-C 18 LS films, leading to extended life-time SERS substrates.

The reproducibility of LS fabrication methodology was also tested from SERS experiments. Three independent plasmonic substrates incorporating AuNP-C18_1:5 LS films were evaluated by means of R6G mapping. Three different SERS mapping in random points were performed for each substrate (area of $250 \mu \mathrm{m} \times 150 \mu \mathrm{m} ; 15 \times 15$ points $\times 3$ mapping $=675$ spectra) and the intensity of the peak at $1510 \mathrm{~cm}^{-1}$ was monitored (Figure 8.b). The results reveal a very good spatial uniformity with an average peak intensity at $1510 \mathrm{~cm}^{-1}$ of $57019 \pm 4930 \mathrm{cts}$ $\mathrm{mW}^{-1} \mathrm{~s}^{-1}$. The average relative standard deviation (RSD) is ca $9 \%$, which is considerably reliable for SERS measurements. This experimental evidence supports the benefits of LS technique for the fabrication of large and homogenous SERS-active regions.

Finally, the theoretical limit of detection (LOD) of the SERS substrates was determined by analysing Figure 8.a. The SERS performance of tightly-packed films of AuNP-C18_1:5 
enables the detection of $\mathrm{R} 6 \mathrm{G}$ in a $10^{-11} \mathrm{M}$ concentration, two orders of magnitude lower than the value previously reported of rhodamine in Au@silicon substrate prepared by LS technique [20]. A linear dependence of the SERS intensity with the R6G concentration is observed within the operating window (Figure 8.c). The $10 \mathrm{pM}$ to $1 \mu \mathrm{M}$ region is defined as the quantification region [37], and can be expressed by the empirical formula: $\log I=0.22 \log C+6.12\left(\mathrm{R}^{2}=0.9906\right)$, where $I$ is the normalized SERS intensity at $1510 \mathrm{~cm}^{-1}$ (cts $\mathrm{mW}^{-1} \mathrm{~s}^{-1}$ ) and $C$ is the R6G concentration $\left(\mathrm{mol} \cdot \mathrm{L}^{-1}\right)$. From these data, the theoretical limit of detection (LOD), calculated from the intensity variation of the characteristic Raman band at $1510 \mathrm{~cm}^{-1}$ equivalent to three times the signal noise value, is estimated to be $5.410^{-13} \mathrm{M}$. Importantly, these findings are either in the same order of magnitude or overpass the reported R6G limit of detection values for Aubased SERS substrates prepared by: i) a sacrificial colloidal film template route by chemical deposition and further physical deposition [38], i.e. $10^{-11} \mathrm{M}$; ii) physical deposition on biomimetic superhydrophobic surfaces [39], i.e. $10^{-7} \mathrm{M}$; iii) electrostatic assembly of nanostars [10], i.e. $10^{-9} \mathrm{M}$; or iv) electrochemical deposition-dissolution cycles [40], i.e. $10^{-12} \mathrm{M}$.

\section{Conclusions}

Homogeneous centimeter scale SERS substrates incorporating $27 \mathrm{~nm}$ in diameter octadecylthiolate capped AuNPs have been easily prepared by the Langmuir-Schaefer technique using MilliQ water as subphase. Such simple and low cost approach requires redispersion of thiol capped Au NPs in 1:5 hexane:chloroform solvent and sylanization of the substrate. These LS films exhibit a large surface coverage of well-ordered and tightly-packed AuNPs. The experimentally obtained AEF, $4.3 \times 10^{8}$, enables detection of R6G at concentrations as low as $10^{-11} \mathrm{M}$. The durability and the spatial uniformity of the SERS properties are clearly outstanding. These results demonstrate the potentialities of the LS technique for the fabrication of large SERS-active substrates with homogeneously distributed Raman response and contribute to the development of low cost SERS systems for routine high throughput analysis. This exploratory work paves the way for further studies focused on SERS applications thanks to the versatility of ligand exchange reactions in thiolate stabilized AuNPs.

\section{Conflicts of interest}


Authors declare that there are no conflicts of interest.

\section{Acknowledgements}

P.C. and S.R-R. are grateful for financial assistance from DGA/Fondos FEDER (construyendo Europa desde Aragón) for funding PLATON (E31_17R) research group. M.P.P., R.M. and M.L. are grateful for financial support from MICINN (CTQ2013-49068-C2-1-R, and CTQ201679419-R).

\section{References}

[1] Fleischmann, M., P.J. Hendra, and A.J. McQuillan, Raman spectra of pyridine adsorbed at a silver electrode. Chem. Phys. Lett., 26 (1974) 163-166.

[2] Le Ru, E., E. Blackie, M. Meyer, and P.G. Etchegoin, Surface enhanced Raman scattering enhancement factors: a comprehensive study. J. Phys. Chem. C., 111 (2007) 13794-13803.

[3] Le Ru, E. and P. Etchegoin, Principles of Surface-Enhanced Raman Spectroscopy and Related Plasmonic Effects2009: Elsevier: Oxford, UK.

[4] Kleinman, S.L., R.R. Frontiera, A.-I. Henry, J.A. Dieringer, and R.P. Van Duyne, Creating, characterizing, and controlling chemistry with SERS hot spots. Phys. Chem. Chem. Phys., 15 (2013) 21-36.

[5] Jahn, M., S. Patze, I.J. Hidi, R. Knipper, A.I. Radu, A. Mühlig, S. Yüksel, V. Peksa, K. Weber, T. Mayerhöfer, D. Cialla-May, and J. Popp, Plasmonic nanostructures for surface enhanced spectroscopic methods. Analyst, 141 (2016) 756-793.

[6] Álvarez-Puebla, R.n.A., Effects of the Excitation Wavelength on the SERS Spectrum. J. Phys. Chem. Lett., 3 (2012) 857-866.

[7] Schmidt, M.S., J. Hübner, and A. Boisen, Large Area Fabrication of Leaning Silicon Nanopillars for Surface Enhanced Raman Spectroscopy. Adv. Mater., 24 (2012) OP11OP18.

[8] Park, S.-G., C. Mun, X. Xiao, A. Braun, S. Kim, V. Giannini, S.A. Maier, and D.-H. Kim, Surface Energy-Controlled SERS Substrates for Molecular Concentration at Plasmonic Nanogaps. Adv. Funct. Mater., 27 (2017) 1703376.

[9] Lafuente, M., I. Pellejero, V. Sebastián, M.A. Urbiztondo, R. Mallada, M.P. Pina, and J. Santamaría, Highly sensitive SERS quantification of organophosphorous chemical warfare agents: A major step towards the real time sensing in the gas phase. Sens. Actuators B. Chem., 267 (2018) 457-466.

[10] Su, Q., X. Ma, J. Dong, C. Jiang, and W. Qian, A reproducible SERS substrate based on electrostatically assisted APTES-functionalized surface-assembly of gold nanostars. ACS Appl. Mater. Interfaces, 3 (2011) 1873-1879.

[11] Fang, Y., N.-H. Seong, and D.D. Dlott, Measurement of the Distribution of Site Enhancements in Surface-Enhanced Raman Scattering. Science, 321 (2008) 388-392.

[12] Mosier-Boss, P.A., Review of SERS Substrates for Chemical Sensing. Nanomaterials, 7 (2017) 142. 
[13] Sharma, B., R.R. Frontiera, A.-I. Henry, E. Ringe, and R.P. Van Duyne, SERS: Materials, applications, and the future. Materials Today, 15 (2012) 16-25.

[14] Ulman, A., An Introduction to Ultrathin Organic Films: From Langmuir-Blodgett to SelfAssembly1991, San Diego: Academic Press, Inc.

[15] Acharya, S., J.P. Hill, and K. Ariga, Soft Langmuir-Blodgett Technique for Hard Nanomaterials. Adv. Mater., 21 (2009) 2959-2981.

[16] Mahmoud, M.A., D. O'Neil, and M.A. El-Sayed, Hollow and Solid Metallic Nanoparticles in Sensing and in Nanocatalysis. Chem. Mater., 26 (2014) 44-58.

[17] Saha, S., M. Ghosh, B. Dutta, and J. Chowdhury, Silver coated gold nanocolloids entrapped in organized Langmuir-Blodgett Film of stearic acid: Potential evidence of a new SERS active substrate. Appl. Surf. Sci., 362 (2016) 364-373.

[18] Chowdhury, J., S. Saha, and M. Ghosh, Self-assembly of metal nanocolloids entrapped in Langmuir Blodgett films templates: Evidence of efficient SERS sensing platforms. Materials Today: Proceedings 5(2018) 10071-10076.

[19] Tahghighi, M., I. Mannelli, D. Janner, and J. Ignés-Mullol, Tailoring plasmonic response by Langmuir-Blodgett gold nanoparticle templating for the fabrication of SERS substrates. Appl. Surf. Sci., 447 (2018) 416-422.

[20] Pérez-Mayen, L., J. Oliva, A. Torres-Castro, and E. De la Rosa, SERS substrates fabricated with star-like gold nanoparticles for zeptomole detection of analytes. Nanoscale, 7 (2015) 10249-10258.

[21] Koh, C.S.L., H.K. Lee, X. Han, H.Y.F. Sim, and X.Y. Ling, Plasmonic nose: integrating the MOF-enabled molecular preconcentration effect with a plasmonic array for recognition of molecular-level volatile organic compounds. Chem. Com., 54 (2018) 2546-2549.

[22] Brust, M., M. Walker, D. Bethell, D.J. Schiffrin, and R. Whyman, Synthesis of thiolderivatised gold nanoparticles in a two-phase liquid-liquid system. Chem. Com., (1994) 801-802.

[23] Fazio, B., C. D’Andrea, A. Foti, E. Messina, A. Irrera, M.G. Donato, V. Villari, N. Micali, O.M. Maragò, and P.G. Gucciardi, SERS detection of Biomolecules at Physiological $\mathrm{pH}$ via aggregation of Gold Nanorods mediated by Optical Forces and Plasmonic Heating. Sci Rep, 6 (2016) 26952.

[24] Lafuente, M., E.J. Berenschot, R.M. Tiggelaar, R. Mallada, N.R. Tas, and M.P. Pina, 3D Fractals as SERS Active Platforms: Preparation and Evaluation for Gas Phase Detection of G-Nerve Agents. Micromachines, 9 (2018) 60.

[25] Cea, P., S. Martín, A. Villares, D. Möbius, and M.C. López, Use of UV-vis reflection spectroscopy for determining the organization of viologen and viologen tetracyanoquinodimethanide monolayers. J. Phys. Chem. B, 110 (2006) 963-970.

[26] Brust, M., N. Stuhr-Hansen, K. Nørgaard, J.B. Christensen, L.K. Nielsen, and T. Bjørnholm, Langmuir- Blodgett Films of Alkane Chalcogenide (S, Se, Te) Stabilized Gold Nanoparticles. Nano Lett., 1 (2001) 189-191.

[27] Hutchings, G.J., M. Brust, and H. Schmidbaur, Gold-an introductory perspective. Chem. Soc. Rev., 37 (2008) 1759-1765.

[28] Ishida, T., Y. Tachikiri, T. Sako, Y. Takahashi, and S. Yamada, Structural characterization and plasmonic properties of two-dimensional arrays of hydrophobic large gold nanoparticles fabricated by Langmuir-Blodgett technique. Appl. Surf. Sci., 404 (2017) 350-356. 
[29] Hsu, C.-L., K.-H. Wang, C.-H. Chang, W.-P. Hsu, and Y.-L. Lee, Surface modification of gold nanoparticles and their monolayer behavior at the air/water interface. Appl. Surf. Sci., 257 (2011) 2756-2763.

[30] Adamson, A.W. and A.P. Gast, Physical Chemistry of Surfaces. $6^{\text {th }}$ Edition1997, New York: John Wiley \& Sons.

[31] Lazarides, A.A. and G.C. Schatz, DNA-linked metal nanosphere materials: structural basis for the optical properties. J. Phys. Chem. B., 104 (2000) 460-467.

[32] Huang, S., G. Tsutsui, H. Sakaue, S. Shingubara, and T. Takahagi, Experimental conditions for a highly ordered monolayer of gold nanoparticles fabricated by the Langmuir-Blodgett method. J. Vac. Sci. Technol B, 19 (2001) 2045-2049.

[33] Martín, S., L.M. Ballesteros, A. González-Orive, H. Oliva, S. Marqués-González, M. Lorenzoni, R. Nichols, F. Pérez-Murano, P.J. Low, and P. Cea, Towards a metallic top contact electrode in molecular electronic devices exhibiting a large surface coverage by photoreduction of silver cations. J. Mater. Chem. C., 4 (2016) 9036-9043.

[34] Magonov, S.N., V. Elings, and M.-H. Whangbo, Phase imagin and stiffness in tappingmode atomic force microscopy. Surf. Sci. , 375 (1997) L384-L391.

[35] Noy, A., C.H. Sanders, D.V. Vezenov, S.S. Wong, and C.M. Lieber, ChemicallySensitive imaging in tapping mode by chemical force microscopy: relationship between phase lag and adhesion. Langmuir, 14 (1998) 1508-1511.

[36] Duan, G., W. Cai, Y. Luo, Z. Li, and Y. Li, Electrochemically induced flowerlike gold nanoarchitectures and their strong surface-enhanced Raman scattering effect. Appl. Phys. Lett., 89 (2006) 211905.

[37] Montes-García, V., B. Gómez-González, D. Martínez-Solís, J.M. Taboada, N. JiménezOtero, J. de Uña-Álvarez, F. Obelleiro, L. García-Río, J. Pérez-Juste, and I. PastorizaSantos, Pillar [5] arene-based supramolecular plasmonic thin films for label-free, quantitative and multiplex SERS detection. ACS Appl. Mater. Interfaces, 9 (2017) 26372-26382.

[38] Zhang, H.H., M. Liu, F. Zhou, D.L. Liu, G.Q. Liu, G.T. Duan, W.P. Cai, and Y. Li, Physical Deposition Improved SERS Stability of Morphology Controlled Periodic Micro/Nanostructured Arrays Based on Colloidal Templates. Small, 11 (2015) 844-853.

[39] Shi, G.C., M.L. Wang, Y.Y. Zhu, L. Shen, W.L. Ma, Y.H. Wang, and R.F. Li, Dragonfly wing decorated by gold nanoislands as flexible and stable substrates for surface-enhanced Raman scattering (SERS). Sci. Rep., 8 (2018).

[40] Mai, F.D., T.C. Hsu, Y.C. Liu, K.H. Yang, and B.C. Chen, A new strategy to prepare surface-enhanced Raman scattering-active substrates by electrochemical pulse deposition of gold nanoparticles. Chem. Com., 47 (2011) 2958-2960. 


\section{List of captions:}

Figure 1. Scheme showing the protocol followed in this contribution: (a) preparation of the gold nanoparticles dispersion; (b) fabrication of Langmuir films and Langmuir-Schaefer modified substrates incorporating octadecylthiolate capped gold nanoparticles (AuNP-C18); (c) application of these substrates for SERS detection.

Figure 2. Surface Pressure vs. area isotherms of AuNP-C18 recorded at $20{ }^{\circ} \mathrm{C}$. BAM images for the AuNP-C18_1:5 films at the indicated surface pressures are shown. The field of view along the $\mathrm{x}$ axes for the BAM images is $1.67 \mathrm{~cm}$.

Figure 3. UV-vis reflection spectra of AuNP-C18_1:5 Langmuir films at the air-water interface at 1 and $20 \mathrm{mN} \cdot \mathrm{m}^{-1}$ (red spectra). Absorption spectra of a dispersion of AuNP-C18_1:5 (blue, straight line) and the absorption spectra of an AuNP-C18_1:5 LS film (blue, dashed line).

Figure 4. SEM images of LS films incorporating AuNP-C18 from different dispersions at 20 $\mathrm{mN} \cdot \mathrm{m}^{-1}$. The field of view along the $x$ axis for the main SEM images is $3 \mu \mathrm{m}$ and $1 \mu \mathrm{m}$ for the inset figures.

Figure 5. SEM images of several LS films incorporating AuNP-C $18 \_1: 5$ at $20 \mathrm{mN} \cdot \mathrm{m}^{-1}$. The field of view along the $x$ axis for the main SEM images is $1500 \mathrm{~nm}$ and $300 \mathrm{~nm}$ for the inset figures.

Figure 6. (a) 1 x $1 \mu \mathrm{m}^{2}$ AFM image of a LS film prepared from an AuNP-C18_1:5 dispersion at $20 \mathrm{mN} \cdot \mathrm{m}^{-1}$. (b) Cross section analysis from the indicated region indicated with a white line in (a). (c) Phase AFM image of a 1 x $1 \mu \mathrm{m}^{2}$ region of a LS film prepared from an AuNP-C18_1:5 dispersion.

Figure 7. SERS mapping obtained at $1510 \mathrm{~cm}^{-1}$ for LS films incorporating (A) AuNP-C 18 _1:5 and (B) AuNP-C $18 \_1: 7$ as well as the average SERS spectrum of R6G $10^{-6} \mathrm{M}$ obtained in each mapping.

Figure 8. (a) SERS spectra measured from AuNP-C18_1:5 LS films after being immersed in $10^{-6}$ $\mathrm{M}, 10^{-8} \mathrm{M}, 10^{-9} \mathrm{M}$ and $10^{-11} \mathrm{M}$ R6G solutions and subsequently air-dried. The SERS spectrum from an aged (sixteen months) AuNP-C18_1:5 LS film immersed in a 10-6 M R6G solution and air-dried is also shown. (b) Average intensity of the peak at $1510 \mathrm{~cm}^{-1}$ of R6G $\left(10^{-6} \mathrm{M}\right)$ of four 
substrates incorporating AuNP-C18_1:5 LS films. Substrates 1 to 3 were measured freshly made and the "aged substrate" was studied 16 months after its fabrication. (c) SERS intensity at 1510 $\mathrm{cm}^{-1}$ as a function of R6G concentration. Each point is result of averaging more than 600 spectra (error bars indicate standard deviation). The dashed line is the linear fitting in the quantitative detection region. 
$\begin{array}{lll}\text { (a) Ligand exchange } & \text { (b) Langmuir-Schaefer films } \quad \text { (c) SERS substrate }\end{array}$

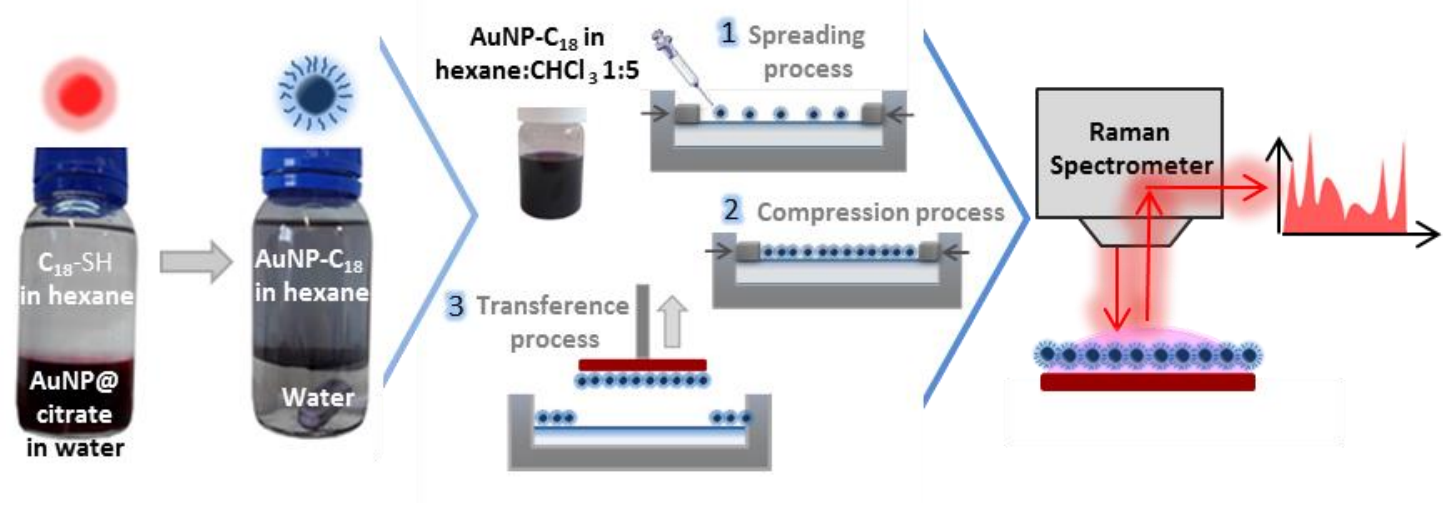

Figure 1. Scheme showing the protocol followed in this contribution: (a) preparation of the gold nanoparticles dispersion; (b) fabrication of Langmuir films and Langmuir-Schaefer modified substrates incorporating octadecylthiolate capped gold nanoparticles (AuNP-C18); (c) application of these substrates for SERS detection. 


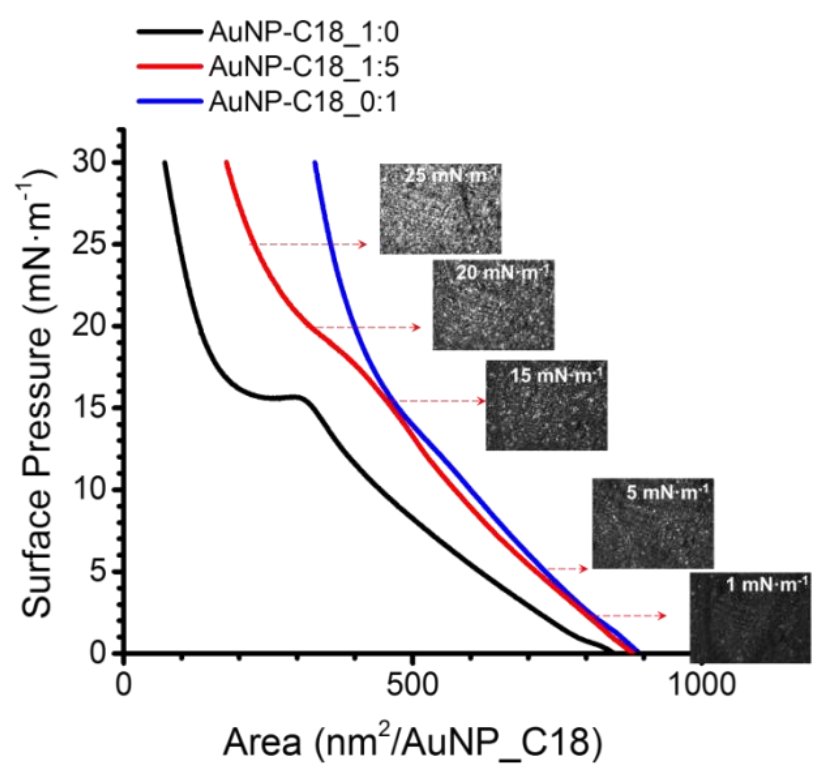

Figure 2. Surface Pressure vs. area isotherms of AuNP-C18 recorded at $20^{\circ} \mathrm{C}$. BAM images for the AuNP-C $\mathrm{C}_{18} 1: 5$ films at the indicated surface pressures are shown. The field of view along the x axes for the BAM images is $1.67 \mathrm{~cm}$. 


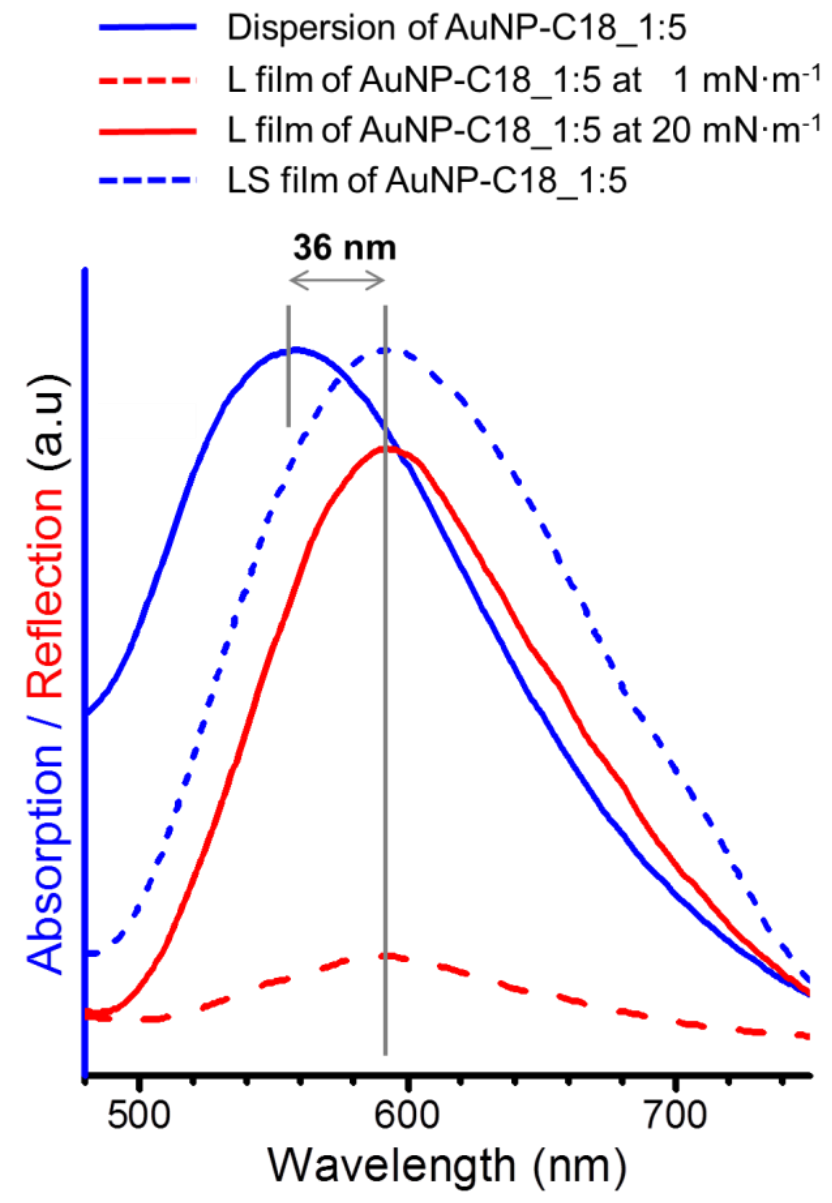

Figure 3. $U V$-vis reflection spectra of AuNP-C $C_{18}$ 1:5 Langmuir films at the air-water interface at 1 and $20 \mathrm{mN} \cdot \mathrm{m}^{-1}$ (red spectra). Absorption spectra of a dispersion of AuNP-C $C_{18}{ }_{1} 1: 5$ (blue, straight line) and the absorption spectra of

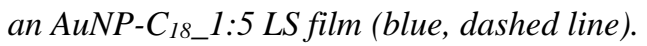



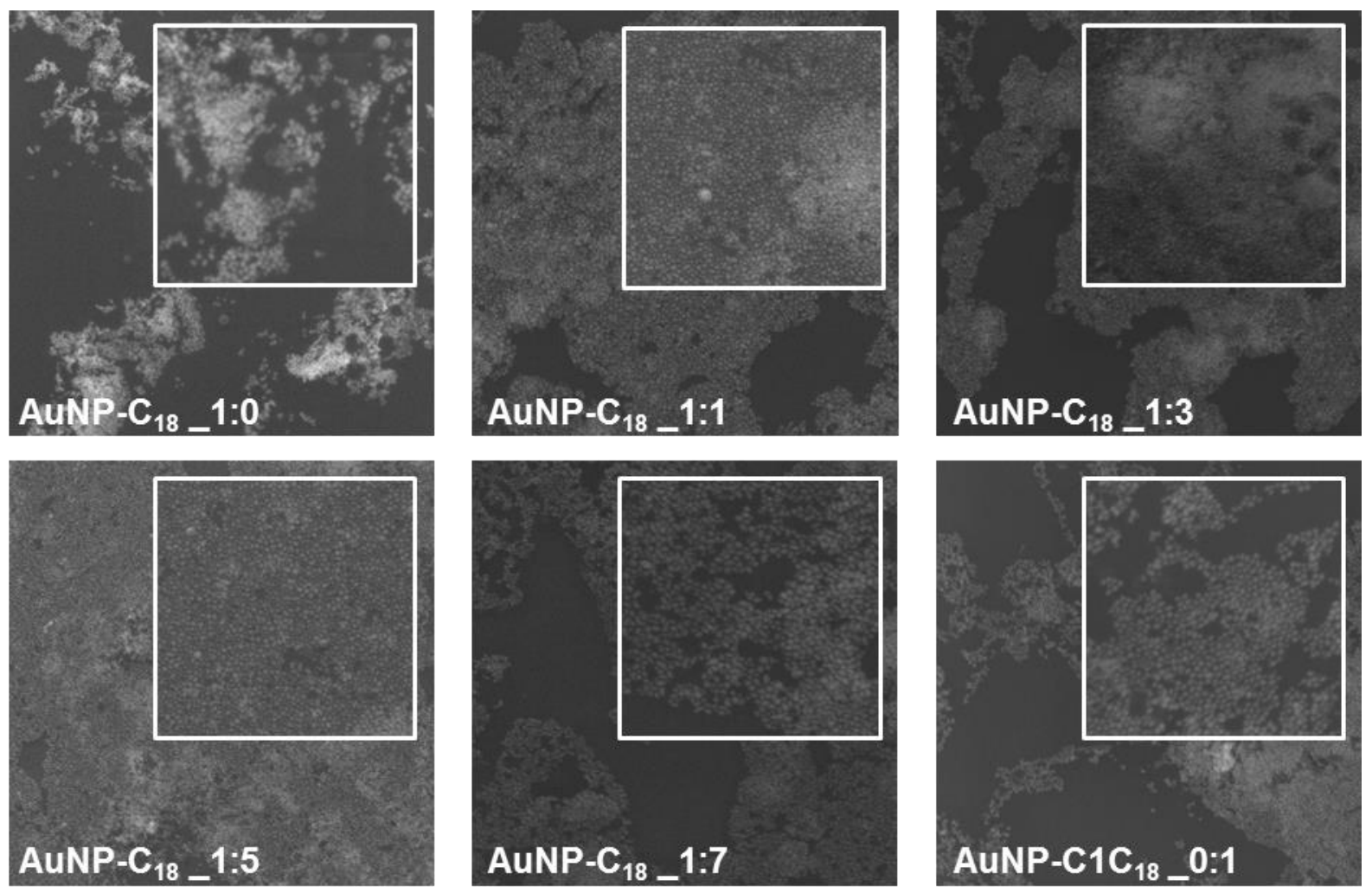

Figure 4. SEM images of LS films incorporating AuNP-C18 from different dispersions at $20 \mathrm{mN} \cdot \mathrm{m}^{-1}$. The field of view along the $x$ axis for the main SEM images is $3 \mu \mathrm{m}$ and $1 \mu \mathrm{m}$ for the inset figures. 


\section{REPRODUCIBILITY}

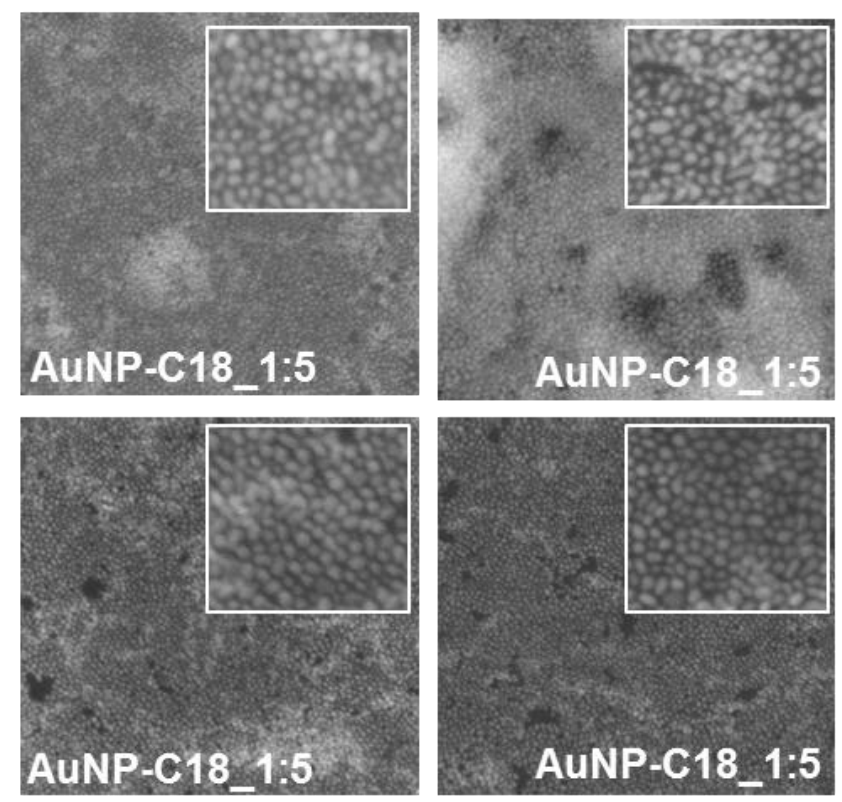

Figure 5. SEM images of several LS films incorporating AuNP-C $C_{18 \_} 1: 5$ at $20 \mathrm{mN} \cdot \mathrm{m}^{-1}$. The field of view along the $x$ axis for the main SEM images is $1500 \mathrm{~nm}$ and $300 \mathrm{~nm}$ for the inset figures. 

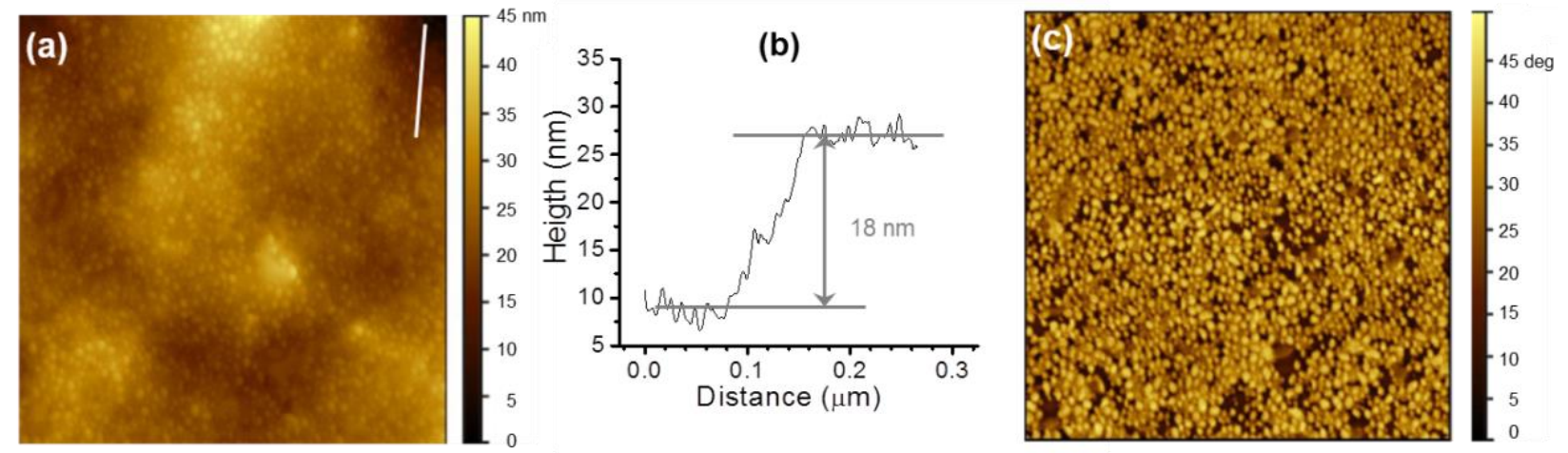

Figure 6. (a) $1 \times 1 \mu \mathrm{m}^{2}$ AFM image of a LS film prepared from an AuNP-C18_1:5 dispersion at $20 \mathrm{mN} \cdot \mathrm{m}^{-1}$. (b) Cross section analysis from the indicated region indicated with a white line in (a). (c) Phase AFM image of a 1 x 1 $\mu m^{2}$ region of a LS film prepared from an AuNP-C18_1:5 dispersion. 

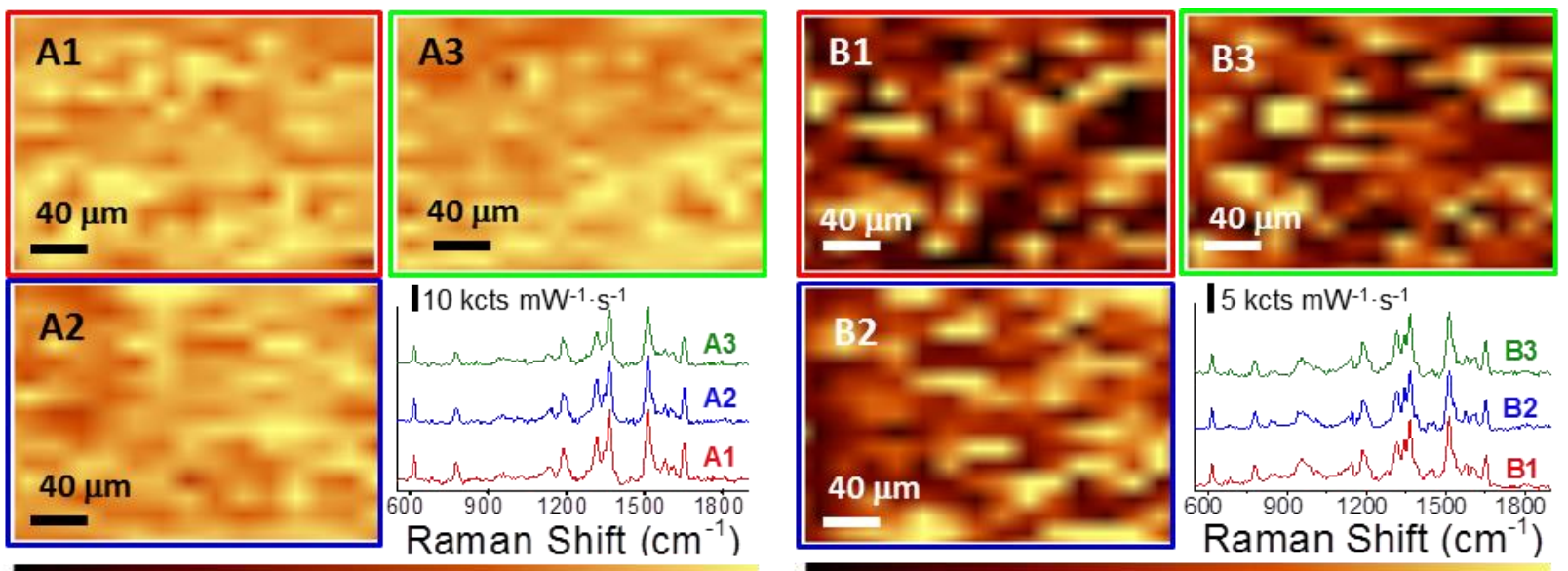

I $5 \mathrm{kcts} \mathrm{mW}^{-1} \cdot \mathrm{s}^{-1}$

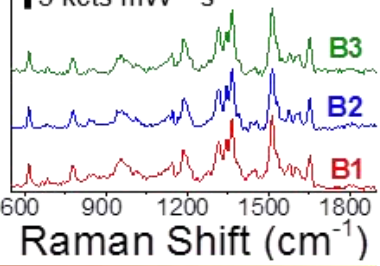

$70 \mathrm{k}$

$30 \mathrm{k}$

Figure 7. SERS mapping obtained at $1510 \mathrm{~cm}^{-1}$ for LS films incorporating (A) AuNP-C $C_{18} \_1: 5$ and (B) AuNP-C 18 _1:7 as well as the average SERS spectrum of R6G $10^{-6} \mathrm{M}$ obtained in each mapping. 


\section{(c) LIMIT OF DETECTION}

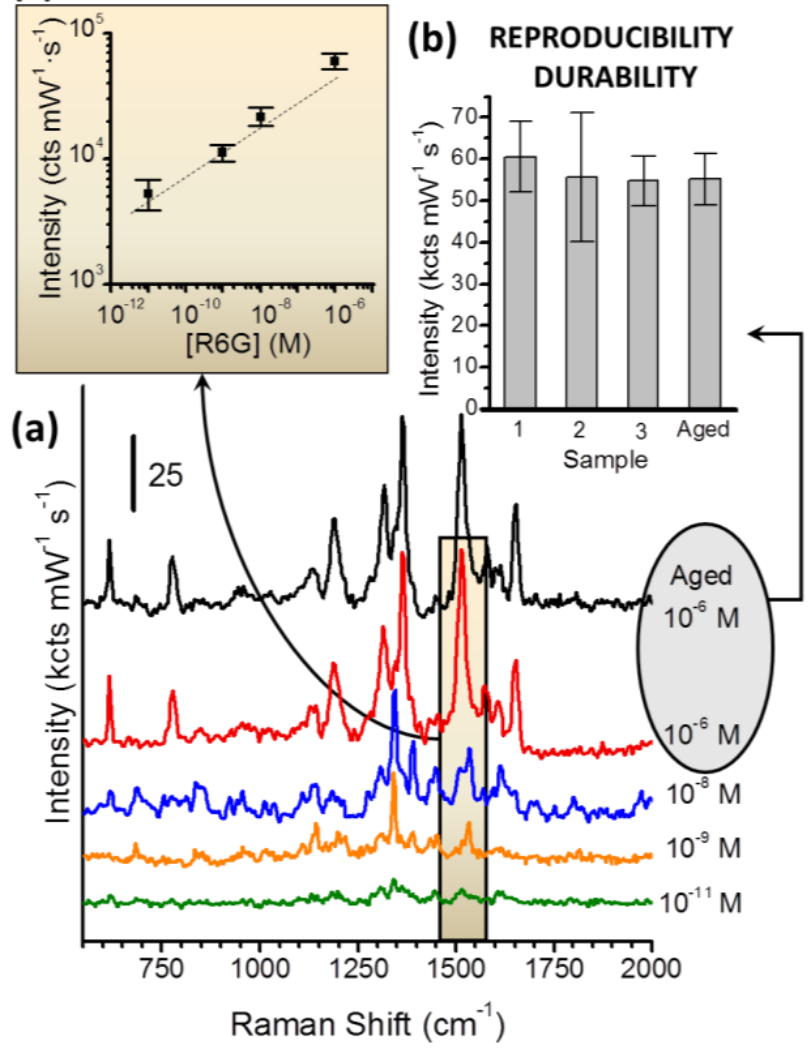

Figure 8. (a) SERS spectra measured from AuNP-C $C_{18} 1: 5 \mathrm{LS}$ films after being immersed in $10^{-6} \mathrm{M}, 10^{-8} \mathrm{M}, 10^{-9} \mathrm{M}$ and $10^{-11} M R 6 G$ solutions and subsequently air-dried. The SERS spectrum from an aged (sixteen months) AuNP-

$C_{18}$ 1:5 LS film immersed in a $10^{-6} \mathrm{M} R 6 \mathrm{G}$ solution and air-dried is also shown. (b) Average intensity of the peak at

$1510 \mathrm{~cm}^{-1}$ of R6G $\left(10^{-6} \mathrm{M}\right)$ of four substrates incorporating AuNP-C18_1:5 LS films. Substrates 1 to 3 were measured freshly made and the "aged substrate" was studied 16 months after its fabrication. (c) SERS intensity at $1510 \mathrm{~cm}^{-1}$ as a function of $R 6 G$ concentration. Each point is result of averaging more than 600 spectra (error bars indicate standard deviation). The dashed line is the linear fitting in the quantitative detection region. 
Graphical Abstract

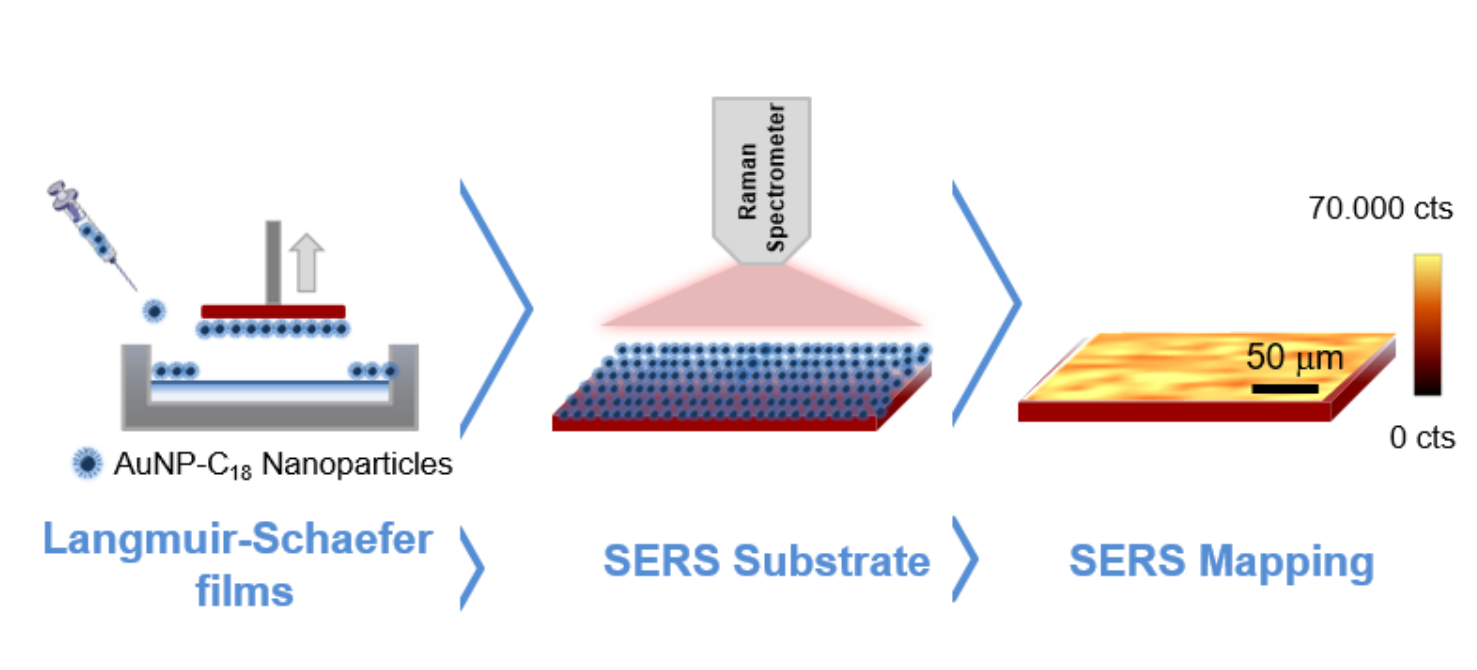




\section{Supporting information}

\section{Towards the reproducible fabrication of homogeneous SERS substrates by Langmuir-Schaefer technique: a low cost and scalable approach for practical SERS based sensing applications}

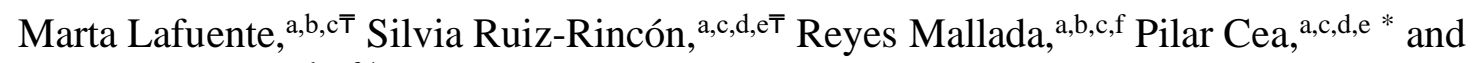
Maria Pilar Pina ${ }^{\mathrm{a}, \mathrm{b}, \mathrm{c}, \mathrm{f}}$ *

anstituto de Nanociencia de Aragón (INA). Campus Rio Ebro, Universidad de Zaragoza, C/Mariano Esquillor, s/n, 50018 Zaragoza, Spain.

bDepartamento de Ingeniería Química y Tecnología del Medio Ambiente, Universidad de Zaragoza, 50009, Spain.

'Instituto de Ciencia de Materiales de Aragón (ICMA), Universidad de Zaragoza-CSIC, 50009 Zaragoza, Spain.

dDepartamento de Química Física, Facultad de Ciencias, Universidad de Zaragoza, 50009, Zaragoza, Spain.

eLaboratorio de Microscopias Avanzadas (LMA). Campus Río Ebro, Universidad de Zaragoza,

C/Mariano Esquilor, s/n, 50018 Zaragoza, Spain.

${ }_{\mathrm{f}}^{\mathrm{f}}$ Networking Research Center on Bioengineering, Biomaterials and Nanomedicine, CIBER-BBN, 28029 Madrid, Spain

$\overline{\mathrm{T}}$ These authors contributed equally to this work

Corresponding authors: pcea@unizar.es (P.C.) and mapina@unizar.es (M.P.P.) 


\section{TABLE OF CONTENTS}

- TEM images of the AuNPs used in this contribution

- Illustration of the chemical structure of the octadecylthiolate-protected AuNPs

- UV-vis spectra of the AuNPs capped with citrate and with

- octadecylthiolate AuNP_C18 behavior at the air water interface as a function of the

- hexane:chloroform spreading solvent ratio

- SERS characterization of films incorporating AuNP_C18_1:3

Characterization of an AuNPs substrate prepared by electrostatic interaction between citrate capped $\mathrm{AuNPs}$ and $\mathrm{SiO}_{2} / \mathrm{Si}$ surface previously modified with a cationic polymer 
- TEM images of the AuNPs used in this contribution
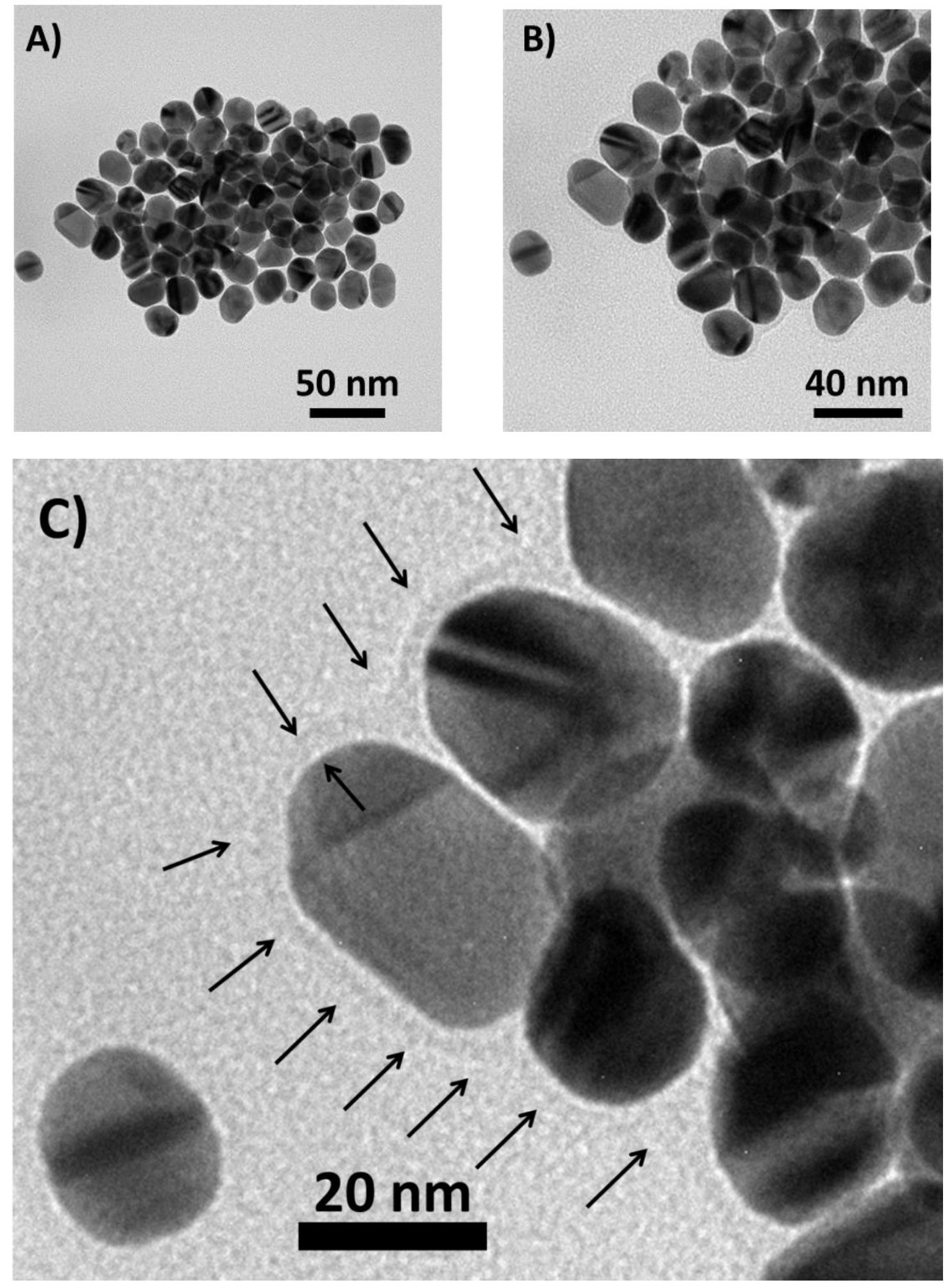

Figure S1. TEM images of (A) AuNPs and (B) AuNP-C 18 where the octadecylthiolate stabilizing layer is visible. (C) magnification of (B) for a better observation of the octadecylthiolate layer. 
- Illustration of the chemical structure of the octadecylthiolate-protected AuNPs

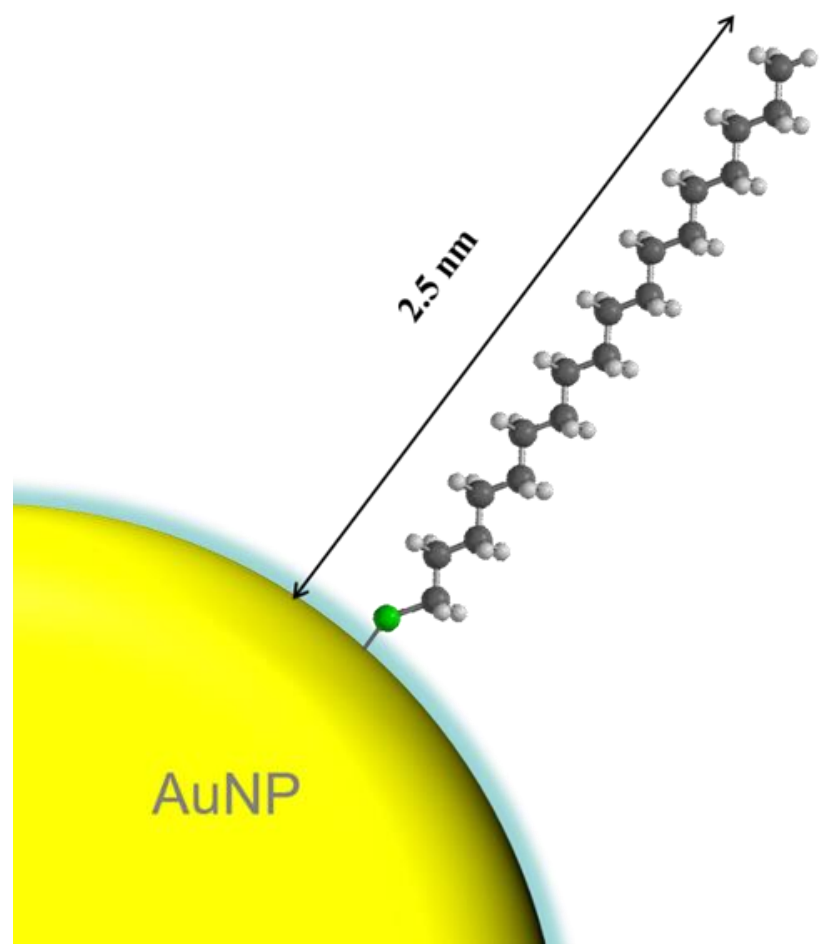

Figure S2. Scheme showing the chemical structure of the octadecylthiolate-protected AuNPs (assuming a trans-zigzag conformation of the alkyl chain) as calculated by Spartan ${ }^{\circledR 08}$ V 1.0.0. 
- UV-vis spectra of the AuNPs capped with citrate and with octadecylthiolate

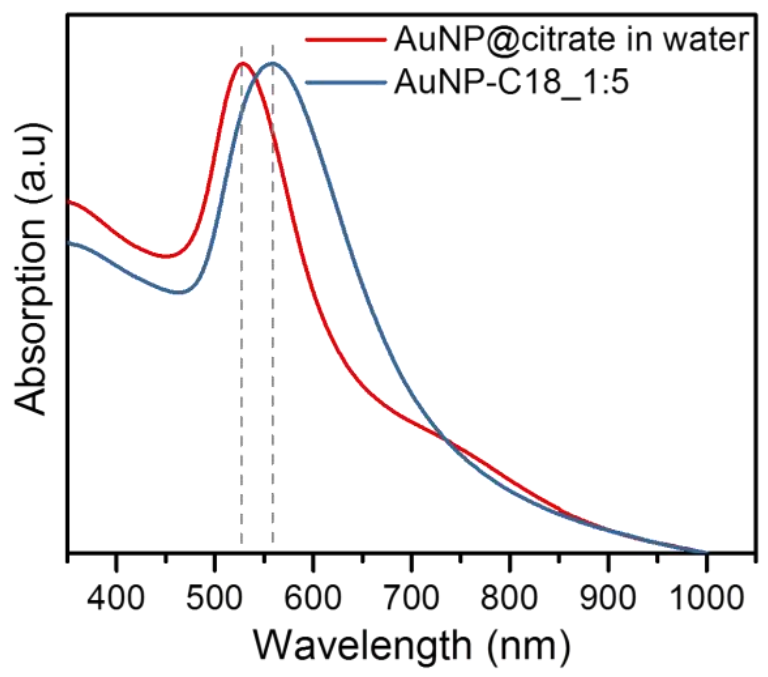

Figure S3. UV-vis spectrum of the AuNPs capped with citrate and dispersed in Milli-Q water and UV-vis spectrum of the AuNPs capped with octadecanethiol and dispersed in a hexane:chloroform 1:5 solution.

- AuNPs_C18 behavior at the air water interface as a function of the hexane:chloroform spreading solvent ratio

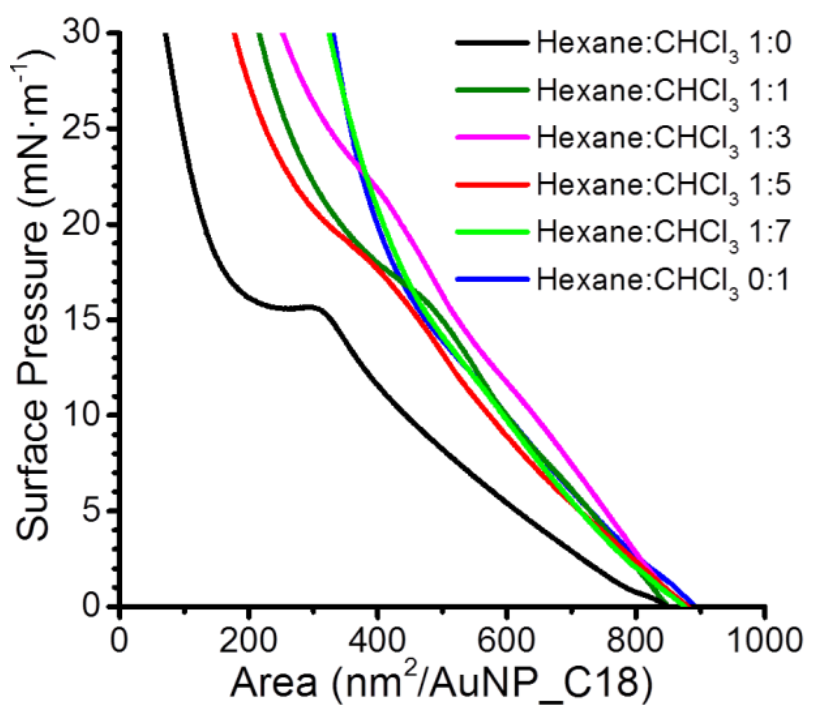

Figure S4. Surface pressure vs. area per gold nanoparticle for the indicated mixtures of spreading solvents. 
- SERS characterization of films incorporating AuNP-C $\mathrm{C}_{18 \_1: 3}$
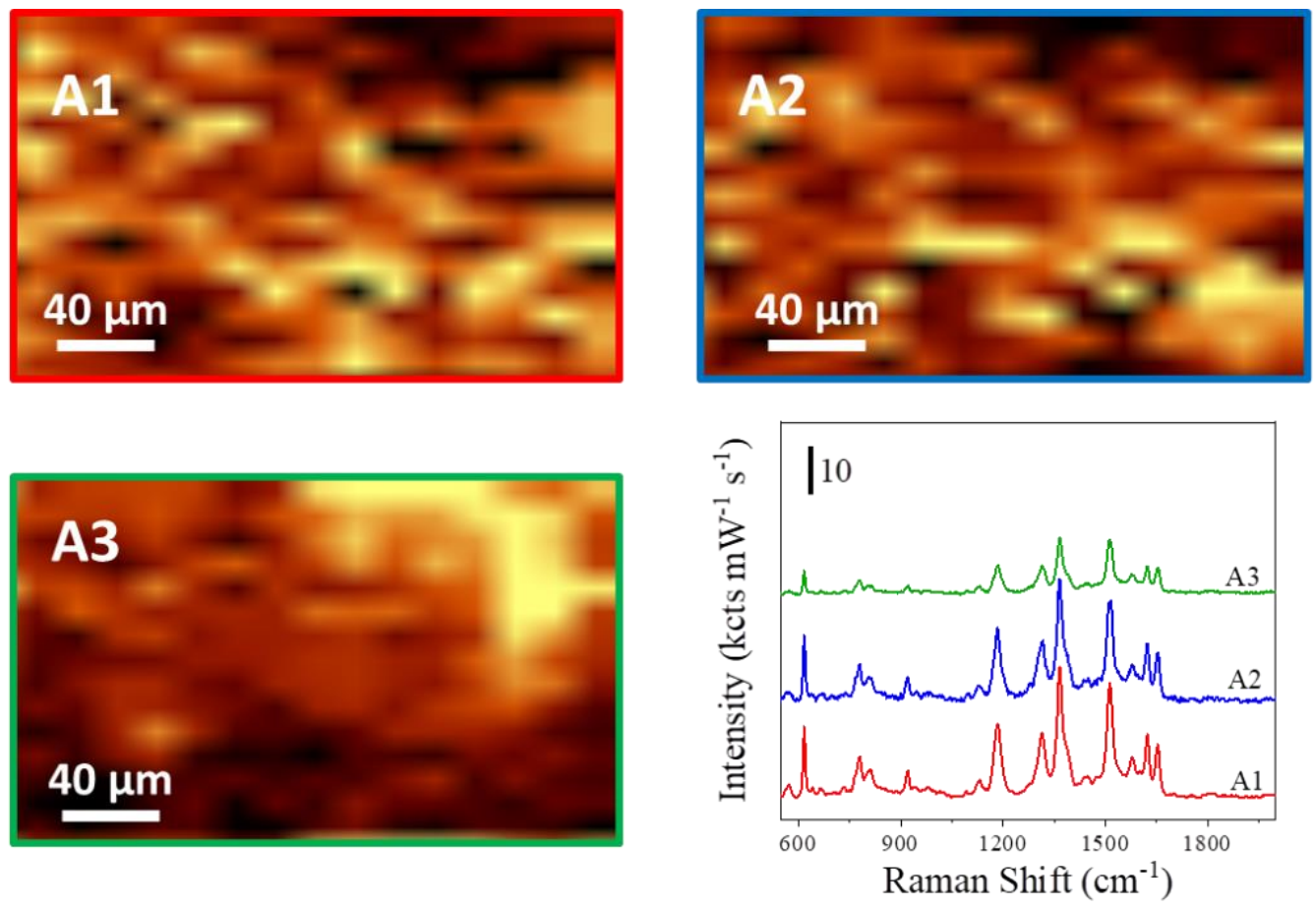

\section{$30 \mathrm{k}$}

Figure S5. SERS mappings obtained at $1510 \mathrm{~cm}^{-1}$ for LS films incorporating AuNP-C $\mathrm{C}_{18} 1: 3$ and the average SERS spectrum of R6G $10^{-6} \mathrm{M}$ obtained in each mapping. 
- Characterization of an AuNPs substrate prepared by electrostatic interaction between citrate capped AuNPs and $\mathrm{SiO}_{2} / \mathrm{Si}$ surface previously modified with a cationic polymer
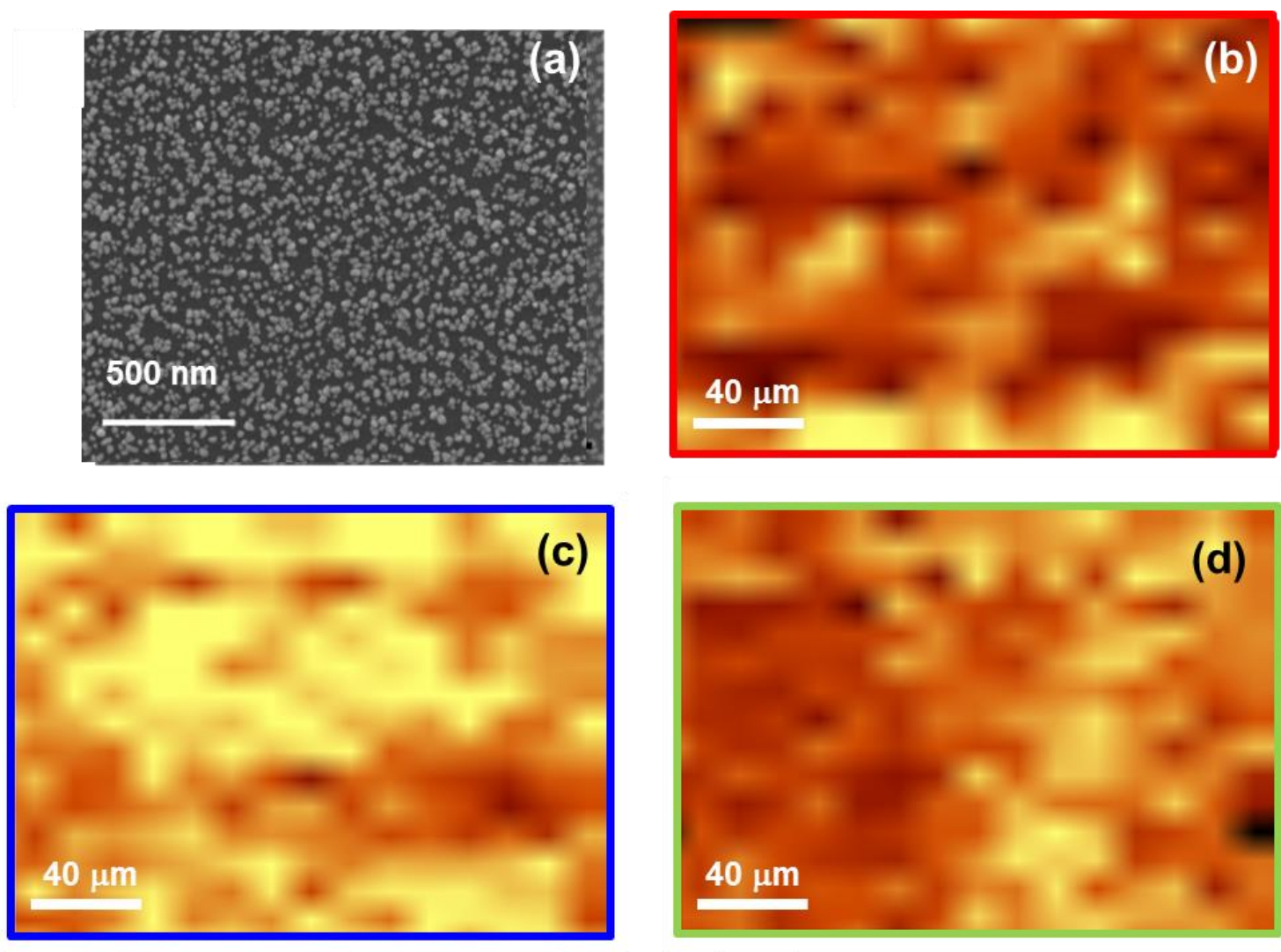

(c)
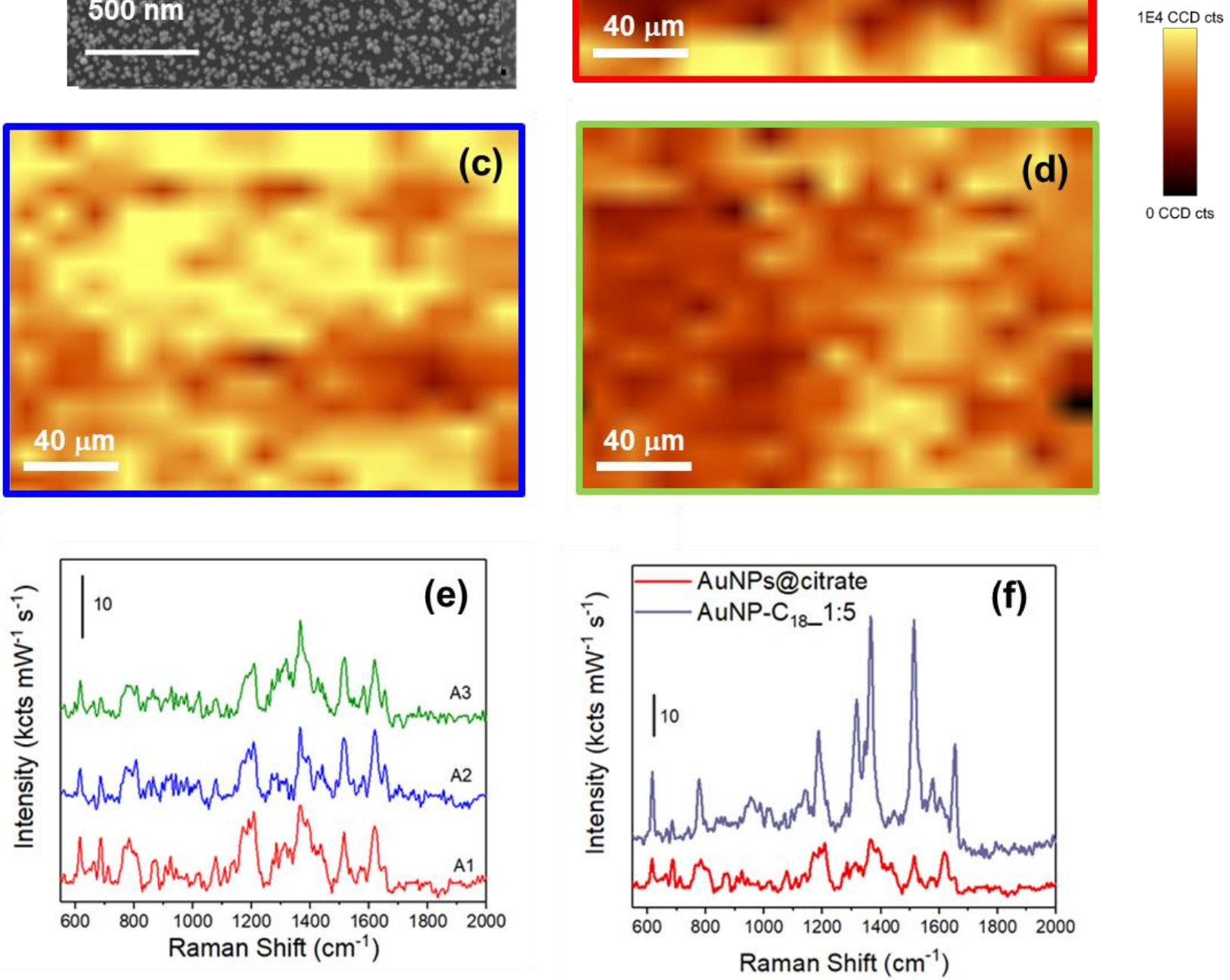

Figure S6. Characterization of an AuNPs substrate prepared by electrostatic interaction between citrate capped AuNPs and $\mathrm{SiO}_{2} / \mathrm{Si}$ surface previously modified with a cationic polyelectrolyte: (a) SEM image; (b-d) SERS mapping obtained at $1510 \mathrm{~cm}^{-1}$; (e) the average SERS spectrum of R6G $1 \mu \mathrm{M}$ obtained in each mapping and (f) the average SERS spectrum of R6G $1 \mu \mathrm{M}$ on AuNPs@citrate monolayers compared with the average SERS spectrum obtained over AuNP-C $18 \_1: 5$ LB films. 
